\title{
Integrable Systems of Neumann Type
}

\author{
Alina Dobrogowska - Tudor S. Ratiu
}

Received: 9 March 2012 / Published online: 29 August 2013

C) Springer Science+Business Media New York 2013

\begin{abstract}
We construct families of integrable systems that interpolate between $N$-dimensional harmonic oscillators and Neumann systems. This is achieved by studying a family of integrable systems generated by the Casimir functions of the Lie algebra of real skew-symmetric matrices and a certain deformation thereof. Involution is proved directly, since the standard involution theorems do not apply to these families. It is also shown that the integrals are independent.
\end{abstract}

Keywords Integrable system · Casimir function · Integrals in involution · Independence . Skew-symmetric matrices · Lie algebra deformation

Mathematics Subject Classification $\quad 37 \mathrm{~J} 35 \cdot 53 \mathrm{D} 17$

\section{Introduction}

The theory of integrable systems is a time-honored subject going back to the founders of theoretical mechanics. They are very important because, in principle, the differential or partial differential equations describing them can be solved and because they are key systems around which many other dynamic and geometric phenomena can be studied, such as perturbations,

To the memory of Klaus Kirchgässner, mentor and friend.

A. Dobrogowska

Section de Mathématiques, École Polytechnique Fédérale de Lausanne, 1015Lausanne, Switzerland

A. Dobrogowska Institute of Mathematics, University of Białystok, Lipowa 41, 15-424Bialystok, Poland e-mail: alaryzko@alpha.uwb.edu.pl

T. S. Ratiu ( $\bigotimes)$

Section de Mathématiques and Bernoulli Center, École Polytechnique Fédérale de Lausanne, 1015Lausanne, Switzerland

e-mail: tudor.ratiu@epfl.ch 
bifurcations, stability, numerics. In addition, integrability for systems on symplectic or Poisson manifolds is characterized by the Liouville-Mineur-Arnold Theorem (see, e.g., [1,4,5]) which states that on a generic leaf of the Poisson manifold, the given Hamiltonian system has half the dimension many integrals that Poisson commute and whose differentials are linearly independent on an open dense set. Many examples of integrable systems are known and have been studied in detail, both in finite and infinite dimensions. The usual approach is to prove involution of the integrals by means of some general involution theorem (such as the Adler-Kostant-Symes theorem [2,7,18], the $R$-matrix method [17], the method of shifted invariants $[10,11]$, the Thimm method $[6,19]$, or by the use of bi-Hamiltonian structures $[9,16]$. If these methods do not apply, reduction methods could be employed (for example, in the Calogero and Moser-Sutherland systems [8]), or one is left with the task of a direct proof. The second step in showing that a system is integrable is the proof of the independence of the differentials of the integrals on an open dense set. No general methods are known for this and, with the exception of some algebraic geometric methods that also give the linearization of the flow (see, e.g., [3] and references therein), the proof of independence has to be done on a case by case basis.

In this paper we study a family of systems that interpolate between harmonic oscillators and Neumann systems in arbitrary dimensions on a specific symplectic leaf in the dual of the Lie algebra of skew-symmetric matrices viewed as upper triangular matrices endowed with the "constant coefficient" Poisson bracket. The idea of considering these systems comes from [14] who studied similar systems in the complex setting and for matrices with a different internal structure. It is remarkable that for the systems we consider, even though they are induced from Lie-Poisson systems, the general known involution theorems do not apply to our knowledge, so we give a direct proof of involution. It turns out that we need two families of functions in order to build a complete set of integrals: these are the usual ones given by traces of powers and a new family of quadratic integrals. We consider two families of integrable systems: one induced from the Lie algebra of real skew-symmetric matrices, the other one from a special deformation of this Lie algebra. The generic independence of the differentials of the integrals of motion is proved by a direct verification.

The paper is organized as follows. In Sect. 2 we determine the symplectic leaf of the "constant coefficient" bracket in the space of upper triangular matrices. Then we construct the first family of integrable systems, a hierarchy generated by the Casimir functions on the dual of the Lie algebra of skew-symmetric matrices and certain quadratic functions. A very particular case of this family is the harmonic oscillator. In Sect. 3 we construct another hierarchy of functions in involution generated by the Casimir functions on a special Lie algebraic deformation of the space of skew symmetric matrices and the same family of quadratic functions. A special case of these systems is the classical Neumann system. The involution of the integrals of both families is proved in the respective sections. Section 4 is devoted to the proof of independence of their differentials on an open dense set of phase space. Several examples are discussed throughout the paper.

\section{Hierarchy Generated by Casimir Functions of $\mathfrak{s o}(n)$}

\subsection{A Symplectic Leaf}

Let $\mathcal{L}_{+}$be the vector space of strictly upper triangular $(n \times n)$-matrices. Relative to the non-degenerate pairing 


$$
\langle\chi, \rho\rangle:=\operatorname{Tr}(\rho \chi), \quad \rho \in \mathcal{L}_{+}, \quad \chi \in \mathfrak{s o}(n),
$$

the space $\mathcal{L}_{+}$is the dual of the Lie algebra so(n). We shall write a general element $\rho \in \mathcal{L}_{+}$ as

$$
\rho=\left(\begin{array}{cc}
\widetilde{A} & B \\
0 & \widetilde{C}
\end{array}\right)
$$

where $\widetilde{A} \in \mathfrak{g l}(2, \mathbb{R})$ and $\widetilde{C} \in \mathfrak{g l}(n-2, \mathbb{R})$ are strictly upper triangular and $B \in \operatorname{Mat}_{2 \times(n-2)}(\mathbb{R})$ (the vector space of $2 \times(n-2)$ real matrices).

Thus, if $f \in C^{\infty}\left(\mathcal{L}_{+}\right)$, we have

$$
\frac{\partial f}{\partial \rho}=\left(\begin{array}{cc}
\frac{\partial f}{\partial A} & \frac{\partial f}{\partial B} \\
-\frac{\partial f}{\partial B^{\top}} & \frac{\partial f}{\partial C}
\end{array}\right),
$$

where $A=\widetilde{A}-\widetilde{A}^{\top}, C=\widetilde{C}-\widetilde{C}^{\top}$, and $\frac{\partial f}{\partial B^{\top}}=\left(\frac{\partial f}{\partial B}\right)^{\top}$. Having Lie algebra $\mathfrak{s o}(n)$ with dual $\mathcal{L}_{+}$one defines the Lie-Poisson bracket on $C^{\infty}\left(\mathcal{L}_{+}\right)$by

$$
\{f, g\}_{1}=\operatorname{Tr}\left(\rho\left[\frac{\partial f}{\partial \rho}, \frac{\partial g}{\partial \rho}\right]\right), \quad f, g \in C^{\infty}\left(\mathcal{L}_{+}\right) .
$$

For any fixed $\rho_{0} \in \mathcal{L}_{+}$, the Poisson bracket

$$
\{f, g\}_{2}=\operatorname{Tr}\left(\rho_{0}\left[\frac{\partial f}{\partial \rho}, \frac{\partial g}{\partial \rho}\right]\right), \quad f, g \in C^{\infty}\left(\mathcal{L}_{+}\right)
$$

is compatible with the Lie-Poisson bracket. In what follows we shall choose

$$
\rho_{0}:=\left(\begin{array}{cc}
\widetilde{A}_{0} & 0 \\
0 & 0
\end{array}\right)
$$

where the $2 \times 2$ matrix $\widetilde{A}_{0}$ is given by

$$
\widetilde{A}_{0}:=\left(\begin{array}{ll}
0 & 1 \\
0 & 0
\end{array}\right) .
$$

A simple calculation shows that the Poisson bracket (2.5) can be written in the form

$$
\{f, g\}_{2}=\operatorname{Tr}\left(\frac{\partial f}{\partial B^{\top}} A_{0} \frac{\partial g}{\partial B}-\frac{\partial g}{\partial A} A_{0} \frac{\partial f}{\partial A}\right),
$$

where $A_{0}=\widetilde{A}_{0}-\widetilde{A}_{0}^{\top}$. The matrix $\frac{\partial g}{\partial A} A_{0} \frac{\partial f}{\partial A}$ is antisymmetric because $A$ and $A_{0}$ are $(2 \times 2)$ antisymmetric matrices (and these always commute). Therefore,

$$
\{f, g\}_{2}(B)=\operatorname{Tr}\left(\frac{\partial f}{\partial B^{\top}} A_{0} \frac{\partial g}{\partial B}\right) .
$$

Thus, we can think of this bracket as being defined on $C^{\infty}\left(\operatorname{Mat}_{2 \times(n-2)}(\mathbb{R})\right)$, i.e., the injective smooth embedding

$$
\left(\operatorname{Mat}_{2 \times(n-2)}(\mathbb{R}),\{,\}_{2}\right) \ni B \longmapsto \rho=\left(\begin{array}{cc}
\widetilde{A} & B \\
0 & \widetilde{C}
\end{array}\right) \in\left(\mathcal{L}_{+},\{,\}_{2}\right),
$$

with $\widetilde{A} \in \mathfrak{g l}(2, \mathbb{R}), \widetilde{C} \in \mathfrak{g l}(n-2, \mathbb{R})$ strictly upper triangular fixed matrices, is Poisson. Since $A_{0}$ is invertible, the Poisson bracket (2.9) is also invertible, i.e., it is a symplectic form on the 
$2(n-2)$-dimensional vector space $\left(\operatorname{Mat}_{2 \times(n-2)}(\mathbb{R}),\{,\}_{2}\right)$. We have proved hence the first statement in the following result.

Proposition 2.1 The $2(n-2)$-dimensional vector space $\left(\operatorname{Mat}_{2 \times(n-2)}(\mathbb{R}),\{,\}_{2}\right)$ is a symplectic leaf of the Poisson manifold $\left(\mathcal{L}_{+},\{,\}_{2}\right)$. The vectors in $\mathbb{R}^{n-2}$ representing the two lines of the matrix $B$ give global symplectic coordinates.

Proof Let $B \in \operatorname{Mat}_{2 \times(n-2)}(\mathbb{R})$ have rows $\vec{p}=\left(p_{1}, \ldots, p_{n-2}\right), \vec{q}=\left(q_{1}, \ldots, q_{n-2}\right) \in \mathbb{R}^{n-2}$. A direct verification shows that the Poisson bracket of $f, g \in C^{\infty}\left(\operatorname{Mat}_{2 \times(n-2)}(\mathbb{R})\right)$ given by (2.9) has the expression

$$
\{f, g\}_{2}(\vec{p}, \vec{q})=\sum_{i=1}^{n-2}\left(\frac{\partial f}{\partial p_{i}} \frac{\partial g}{\partial q_{i}}-\frac{\partial f}{\partial q_{i}} \frac{\partial g}{\partial p_{i}}\right),
$$

i.e., it is the canonical bracket on $\mathbb{R}^{n-2} \times \mathbb{R}^{n-2}$.

2.2 The First Family of Functions in Involution

Next, we show that the Casimir functions

$$
H_{k}(B):=\operatorname{Tr}\left(\widetilde{\rho}^{2 k}\right), \quad k \in \mathbb{N}, \quad \tilde{\rho}:=\rho-\rho^{\top}=\left(\begin{array}{cc}
A & B \\
-B^{\top} & C
\end{array}\right),
$$

of the Poisson bracket (2.4) are in involution with respect to the Poisson bracket (2.9). Since the derivative of $H_{k}$ is $D H_{k}=2 k \widetilde{\rho}^{2 k-1}$, we get

$$
\begin{aligned}
& \frac{\partial H_{k}}{\partial B}=-4 k P_{+} \widetilde{\rho}^{2 k-1} P_{-}, \\
& \frac{\partial H_{k}}{\partial B^{\top}}=4 k P_{-} \widetilde{\rho}^{2 k-1} P_{+}
\end{aligned}
$$

respectively, where $P_{+}, P_{-}$are the orthogonal projectors given, in block matrix notation, by

$$
P_{+}=\left(\begin{array}{ll}
1 & 0 \\
0 & 0
\end{array}\right), \quad P_{-}=\left(\begin{array}{ll}
0 & 0 \\
0 & 1
\end{array}\right) .
$$

A direct calculation, using $P_{+}+P_{-}=\mathbf{1}, P_{+} P_{-}=\mathbf{0}, P_{+}^{2}=P_{+}$, and $P_{-}^{2}=P_{-}$, yields

$$
\begin{aligned}
\left\{H_{k}, H_{l}\right\}_{2} & =\operatorname{Tr}\left(\frac{\partial H_{k}}{\partial B^{\top}} A_{0} \frac{\partial H_{l}}{\partial B}\right)=-16 k l \operatorname{Tr}\left(P_{-} \widetilde{\rho}^{2 k-1} P_{+} A_{0} P_{+} \widetilde{\rho}^{2 l-1} P_{-}\right) \\
& =-16 k l \operatorname{Tr}\left(\widetilde{\rho}^{k+l-1} P_{+} A_{0} P_{+} \widetilde{\rho}^{k+l-1}\right)+16 k l \operatorname{Tr}\left(P_{+} \widetilde{\rho}^{2 k-1} P_{+} A_{0} P_{+} \widetilde{\rho}^{2 l-1} P_{+}\right) \\
& =0,
\end{aligned}
$$

because the matrix in the first term is antisymmetric and the matrix in the second term, as a product of three antisymmetric $2 \times 2$ matrices, is also antisymmetric.

We obtain a hierarchy of Hamiltonian equations generated by the Hamiltonians $H_{k}, k \in \mathbb{N}$, for the Poisson bracket (2.9), namely

$$
\frac{\partial B}{\partial t_{k}}=A_{0} \frac{\partial H_{k}}{\partial B} .
$$


The Hamiltonians for $k=1,2,3$ have the expressions

$$
\begin{aligned}
H_{1}= & \operatorname{Tr} A^{2}+\operatorname{Tr} C^{2}-2 \operatorname{Tr} B^{\top} B \\
H_{2}= & \operatorname{Tr} A^{4}+\operatorname{Tr} C^{4}-4 \operatorname{Tr} A^{2} B B^{\top}-4 \operatorname{Tr} C^{2} B^{\top} B \\
& +2 \operatorname{Tr} B B^{\top} B B^{\top}-4 \operatorname{Tr} A B C B^{\top} \\
H_{3}= & \operatorname{Tr} A^{6}+\operatorname{Tr} C^{6}-\operatorname{Tr} A^{4} B B^{\top}-6 \operatorname{Tr} C^{4} B^{\top} B \\
& -2 \operatorname{Tr} B^{\top} B B^{\top} B B^{\top} B+6 \operatorname{Tr} A^{2} B B^{\top} B B^{\top}+6 \operatorname{Tr} C^{2} B^{\top} B B^{\top} B- \\
& -6 \operatorname{Tr} A^{3} B C B^{\top}-6 \operatorname{Tr} C^{3} B^{\top} A B-6 \operatorname{Tr} A^{2} B C^{2} B^{\top}+3 \operatorname{Tr} A B B^{\top} A B B^{\top} \\
& +3 \operatorname{Tr} C B^{\top} B C B^{\top} B+\operatorname{Tr} A B B^{\top} B C B^{\top}+\operatorname{Tr} A B C B^{\top} B B^{\top} .
\end{aligned}
$$

For these Hamiltonians, the equations of motion are

$$
\begin{aligned}
\frac{\partial B}{\partial t_{1}}=- & 4 A_{0} B, \\
\frac{\partial B}{\partial t_{2}}=8 & \left(a^{2} A_{0} B-A_{0} B C^{2}+A_{0} B B^{\top} B+a B C\right), \\
\frac{\partial B}{\partial t_{3}}=12 & \left(-a^{4} A_{0} B-A_{0} B C^{4}-A_{0} B B^{\top} B B^{\top} B-2 a^{2} A_{0} B B^{\top} B+\right. \\
& +A_{0} B C^{2} B^{\top} B+A_{0} B B^{\top} B C^{2}-a^{3} B C+a B C^{3}+a^{2} A_{0} B C^{2}- \\
& -a B B^{\top} A B+A_{0} B C B^{\top} B C-a B B^{\top} B C+A_{0} B B^{\top} A B C+ \\
& \left.+A_{0} B C B^{\top} A B-a B C B^{\top} B\right),
\end{aligned}
$$

respectively.

2.3 The Second Family of Functions in Involution

This hierarchy has an extra family of integrals of motion in involution given by

$$
\delta_{k}(B):=\operatorname{Tr}\left(B C^{2 k-1} B^{\top} A_{0}\right), \quad k \in \mathbb{N}
$$

To prove involutivity, note that

$$
\begin{aligned}
\left\{\delta_{k}, \delta_{l}\right\}_{2} & =\operatorname{Tr}\left(\frac{\partial \delta_{k}}{\partial B^{\top}} A_{0} \frac{\partial \delta_{l}}{\partial B}\right) \\
& =4 \operatorname{Tr}\left(\left(A_{0} B C^{2 k-1}\right)^{\top} A_{0}\left(C^{2 l-1} B^{\top} A_{0}\right)^{\top}\right) \\
& =4 \operatorname{Tr}\left(C^{2 k-1} B^{\top} A_{0}^{3} B C^{2 l-1}\right)=-4 \operatorname{Tr}\left(C^{k+l-1} B^{\top} A_{0} B C^{k+l-1}\right)=0,
\end{aligned}
$$

because $A_{0}^{2}=-\mathbf{1}$ and the matrix $C^{k+l-1} B^{\top} A_{0} B C^{k+l-1}$ is antisymmetric.

\subsection{Involution of All Functions}

Finally, we prove that $\delta_{k}$ commutes with $H_{l}$. This will be done in two steps. First, we prove a following recursion formula. Since $C=P_{-} \widetilde{\rho} P_{-}, B^{\top}=-P_{-} \widetilde{\rho} P_{+}$, and $A_{0}^{2}=-\mathbf{1}$, using 
the relations $P_{+}+P_{-}=\mathbf{1}, P_{+}^{2}=P_{+}, P_{-}^{2}=P_{-}$, and $P_{+} P_{-}=\mathbf{0}$, we get for any $k, l \in \mathbb{N}$, $k \geq 2$,

$$
\begin{aligned}
\left\{\delta_{k}, H_{l}\right\}_{2}= & \operatorname{Tr}\left(\frac{\partial \delta_{k}}{\partial B^{\top}} A_{0} \frac{\partial H_{l}}{\partial B}\right)=-8 l \operatorname{Tr}\left(C^{2 k-1} B^{\top} A_{0}^{2} P_{+} \widetilde{\rho}^{2 l-1} P_{-}\right) \\
= & -8 l \operatorname{Tr}\left(\left(P_{-} \widetilde{\rho} P_{-}\right)^{2 k-1} P_{-} \widetilde{\rho} P_{+} \widetilde{\rho}^{2 l-1}\right) \\
= & -8 l \operatorname{Tr}\left(P_{-} \widetilde{\rho} P_{-}\left(P_{-} \widetilde{\rho} P_{-}\right)^{2 k-2} P_{-} \widetilde{\rho} P_{+} \widetilde{\rho}^{2 l-1}\right) \\
= & -8 l \operatorname{Tr}\left(\widetilde{\rho}\left(P_{-} \widetilde{\rho} P_{-}\right)^{2 k-2} P_{-} \widetilde{\rho} P_{+} \widetilde{\rho}^{2 l-1}\right) \\
& +8 l \operatorname{Tr}\left(P_{+} \widetilde{\rho}\left(P_{-} \widetilde{\rho} P_{-}\right)^{2 k-2} P_{-} \widetilde{\rho} P_{+} \widetilde{\rho}^{2 l-1} P_{+}\right) \\
= & -8 l \operatorname{Tr}\left(\left(P_{-} \widetilde{\rho} P_{-}\right)^{2 k-2} P_{-} \widetilde{\rho} P_{+} \widetilde{\rho}^{2 l}\right) \\
& +8 l \operatorname{Tr}\left(\left(P_{-} \widetilde{\rho} P_{-}\right)^{k-1} \widetilde{\rho} P_{+} \widetilde{\rho}^{2 l-1} P_{+} \widetilde{\rho}\left(P_{-} \widetilde{\rho} P_{-}\right)^{k-1}\right) \\
= & -8 l \operatorname{Tr}\left(P_{-} \widetilde{\rho}\left(P_{-} \widetilde{\rho} P_{-}\right)^{2 k-3} \widetilde{\rho} P_{+} \widetilde{\rho}^{2 l}\right) \\
= & -8 l \operatorname{Tr}\left(\widetilde{\rho}\left(P_{-} \widetilde{\rho} P_{-}\right)^{2 k-3} P_{-} \widetilde{\rho} P_{+} \widetilde{\rho}^{2 l}\right)+8 l \operatorname{Tr}\left(P_{+} \widetilde{\rho}\left(P_{-} \widetilde{\rho} P_{-}\right)^{2 k-3} P_{-} \widetilde{\rho} P_{+} \widetilde{\rho}^{2 l} P_{+}\right) \\
= & -8 l \operatorname{Tr}\left(\left(P_{-} \widetilde{\rho} P_{-}\right)^{2(k-1)-1} P_{-} \widetilde{\rho} P_{+} \widetilde{\rho}^{2(l+1)-1}\right) \\
& +8 l \operatorname{Tr}\left(\left(P_{+} \widetilde{\rho} P_{-}\right)\left(P_{-} \widetilde{\rho} P_{-}\right)^{2 k-3}\left(P_{-} \widetilde{\rho} P_{+}\right)\left(P_{+} \widetilde{\rho}^{2 l} P_{+}\right)\right) \\
= & -8 l \operatorname{Tr}\left(C^{2(k-1)-1} B^{\top} A_{0}^{2} P_{+} \widetilde{\rho}^{2(l+1)-1} P_{-}\right)=\frac{l}{l+1}\left\{\delta_{k-1}, H_{l+1}\right\}_{2} .
\end{aligned}
$$

because $\left(P_{+} \widetilde{\rho} P_{-}\right)\left(P_{-} \widetilde{\rho} P_{-}\right)^{2 k-3}\left(P_{-} \widetilde{\rho} P_{+}\right)$is an antisymmetric $2 \times 2$ matrix and $P_{+} \widetilde{\rho}^{2 l} P_{+}$ is a symmetric $2 \times 2$ matrix. Note that many times we used the fact that a trace is invariant under cyclic permutations and the matrix $\left(P_{-} \widetilde{\rho} P_{-}\right)^{n-1} \widetilde{\rho} P_{+} \widetilde{\rho}^{2 m-1} P_{+} \widetilde{\rho}\left(P_{-} \widetilde{\rho} P_{-}\right)^{n-1}$ is antisymmetric. Second, we show that $\delta_{1}$ commutes with $H_{l}$ :

$$
\begin{aligned}
\left\{\delta_{1}, H_{l}\right\}_{2} & =-8 l \operatorname{Tr}\left(C B^{\top} A_{0}^{2} P_{+} \widetilde{\rho}^{2 l-1} P_{-}\right)=-8 l \operatorname{Tr}\left(P_{-} \widetilde{\rho} P_{-} \widetilde{\rho} P_{+} \widetilde{\rho}^{2 l-1}\right) \\
& =-8 l \operatorname{Tr}\left(\widetilde{\rho} P_{-} \widetilde{\rho} P_{+} \widetilde{\rho}^{2 l-1}\right)+8 l \operatorname{Tr}\left(P_{+} \widetilde{\rho} P_{-} \widetilde{\rho} P_{+} \widetilde{\rho}^{2 l-1} P_{+}\right) \\
& =-8 l \operatorname{Tr}\left(P_{-} \widetilde{\rho} P_{+} \widetilde{\rho}^{2 l}\right)-8 l \operatorname{Tr}\left(\left(P_{-} \widetilde{\rho} P_{+}\right) \widetilde{\rho}^{2 l-1}\left(P_{-} \widetilde{\rho} P_{+}\right)^{\top}\right) \\
& =-8 l \operatorname{Tr}\left(\widetilde{\rho} P_{+} \widetilde{\rho}^{2 l}\right)+8 l \operatorname{Tr}\left(\left(P_{+} \widetilde{\rho} P_{+}\right)\left(P_{+} \widetilde{\rho}^{2 l} P_{+}\right)\right) \\
& =-8 l \operatorname{Tr}\left(P_{+} \widetilde{\rho}^{2 l+1} P_{+}\right)=0,
\end{aligned}
$$

because $\left(P_{-} \widetilde{\rho} P_{+}\right) \widetilde{\rho}^{2 l-1}\left(P_{-} \widetilde{\rho} P_{+}\right)^{\top}$ is antisymmetric, $P_{+} \widetilde{\rho} P_{+}$is antisymmetric and $P_{+} \widetilde{\rho}^{2 l} P_{+}$ is symmetric, and $P_{+} \widetilde{\rho}^{2 l+1} P_{+}$is antisymmetric.

Formulas (2.24) and (2.25) immediately imply that $\left\{\delta_{k}, H_{l}\right\}_{2}=0$ for any $k, l \in \mathbb{N}$. Thus the family of functions $\left\{H_{l}, \delta_{k} \mid k, l \in \mathbb{N}\right\}$ are in involution.

The candidates for the independent integrals depend on whether $n$ is even or odd. If $n=$ $2 p+1$, we take $H_{1}, \ldots, H_{p}, \delta_{1}, \ldots, \delta_{p-1}$ as the system of integrals in involution. Hence we have $2 p-1=n-2$ integrals in involution. If $n=2 p$, we take $H_{1}, \ldots, H_{p-1}, \delta_{1}, \ldots, \delta_{p-1}$ as the system of integrals in involution. Hence we have $2 p-2=n-2$ integrals in involution. 
Example 2.2 In this example we consider the case when $\widetilde{\rho}$ is $3 \times 3$-matrix and assumes the form

$$
\tilde{\rho}=\left(\begin{array}{ccc}
0 & a & p_{1} \\
-a & 0 & q_{1} \\
-p_{1} & -q_{1} & 0
\end{array}\right)
$$

The Poisson bracket (2.9) becomes

$$
\{f, g\}_{2}\left(p_{1}, q_{1}\right)=\frac{\partial f}{\partial p_{1}} \frac{\partial g}{\partial q_{1}}-\frac{\partial f}{\partial q_{1}} \frac{\partial g}{\partial p_{1}}
$$

for any $f, g \in C^{\infty}\left(\mathbb{R}^{2}\right)$. In this case we have only one Hamiltonian

$$
H_{1}=-2\left(a^{2}+p_{1}^{2}+q_{1}^{2}\right),
$$

which generates Hamilton's equations for the harmonic oscillator

$$
\begin{aligned}
& \frac{\partial p_{1}}{\partial t}=\left\{p_{1}, H_{1}\right\}_{2}=-4 q_{1}, \\
& \frac{\partial q_{1}}{\partial t}=\left\{q_{1}, H_{1}\right\}_{2}=4 p_{1} .
\end{aligned}
$$

Example 2.3 In this example we consider the case when $\tilde{\rho}$ is $4 \times 4$-matrix and assumes the form

$$
\widetilde{\rho}=\left(\begin{array}{cccc}
0 & a & p_{1} & p_{2} \\
-a & 0 & q_{1} & q_{2} \\
-p_{1} & -q_{1} & 0 & c \\
-p_{2} & -q_{2} & -c & 0
\end{array}\right) .
$$

The Poisson bracket (2.9) becomes

$$
\{f, g\}_{2}\left(p_{i}, q_{i}\right)=\frac{\partial f}{\partial p_{1}} \frac{\partial g}{\partial q_{1}}-\frac{\partial f}{\partial q_{1}} \frac{\partial g}{\partial p_{1}}+\frac{\partial f}{\partial p_{2}} \frac{\partial g}{\partial q_{2}}-\frac{\partial f}{\partial q_{2}} \frac{\partial g}{\partial p_{2}}
$$

for $f, g \in C^{\infty}\left(\mathbb{R}^{4}\right)$. The integrals in involution are

$$
\begin{aligned}
H_{1} & =-2\left(a^{2}+c^{2}+p_{1}^{2}+p_{2}^{2}+q_{1}^{2}+q_{2}^{2}\right), \\
\delta_{1} & =2 c\left(p_{2} q_{1}-p_{1} q_{2}\right) .
\end{aligned}
$$

Hamilton's equations for $H_{1}$ give again the equations for the harmonic oscillator.

Since $\widetilde{\rho} \in \mathfrak{s o}(4)$, the last invariant is the Pfaffian (the square root of the determinant), which for the writing (2.31) equals $a c+p_{2} q_{1}-p_{1} q_{2}=a c+\frac{1}{2 c} \delta_{1}$, i.e., the Pfaffian is equal to $\delta_{1}$ up to multiplication and addition with constants. If we take $H_{2}=2\left(a^{2}+c^{2}+p_{1}^{2}+\right.$ $\left.p_{2}^{2}+q_{1}^{2}+q_{2}^{2}\right)^{2}-4\left(a c+p_{2} q_{1}-p_{1} q_{2}\right)^{2}=\frac{1}{2} H_{1}^{2}-\left(2 a c+\frac{1}{c} \delta_{1}\right)^{2}$, the same situation occurs: $\mathrm{H}_{2}$ is a function of $H_{1}$ and $\delta_{1}$.

Example 2.4 In this example we consider the case when $\widetilde{\rho}$ is a $5 \times 5$-matrix and assumes the form

$$
\widetilde{\rho}=\left(\begin{array}{ccccc}
0 & a & p_{1} & p_{2} & p_{3} \\
-a & 0 & q_{1} & q_{2} & q_{3} \\
-p_{1} & -q_{1} & 0 & -c_{3} & c_{2} \\
-p_{2} & -q_{2} & c_{3} & 0 & -c_{1} \\
-p_{3} & -q_{3} & -c_{2} & c_{1} & 0
\end{array}\right)
$$


As usual, (2.9) is the standard Poisson bracket

$$
\{f, g\}_{2}\left(p_{i}, q_{i}\right)=\sum_{i=1}^{3}\left(\frac{\partial f}{\partial p_{i}} \frac{\partial g}{\partial q_{i}}-\frac{\partial f}{\partial q_{i}} \frac{\partial g}{\partial p_{i}}\right)
$$

for $f, g \in C^{\infty}\left(\mathbb{R}^{6}\right)$. The integrals in involution are

$$
\begin{aligned}
& H_{1}=-2\left(a^{2}+\|\vec{C}\|^{2}+\|\vec{p}\|^{2}+\|\vec{q}\|^{2}\right), \\
& H_{2}=\frac{1}{2} H_{1}^{2}-4(\vec{q} \cdot \vec{C})^{2}-4(\vec{p} \cdot \vec{C})^{2}-4\|-a \vec{C}+\vec{q} \times \vec{p}\|^{2}, \\
& \delta_{1}=-2 \vec{C} \cdot(\vec{q} \times \vec{p}),
\end{aligned}
$$

where $\vec{p}=\left(p_{1}, p_{2}, p_{3}\right), \vec{q}=\left(q_{1}, q_{2}, q_{3}\right)$, and $\vec{C}=\left(c_{1}, c_{2}, c_{3}\right)$. Hamilton's equations for $\mathrm{H}_{2}$ are

$$
\begin{aligned}
& \frac{\partial \vec{p}}{\partial t}=8\left(-\frac{1}{2} H_{1} \vec{q}-(\vec{q} \cdot \vec{C}) \vec{C}+\vec{p} \times(\vec{p} \times \vec{q})-a \vec{C} \times \vec{p}\right), \\
& \frac{\partial \vec{q}}{\partial t}=8\left(\frac{1}{2} H_{1} \vec{p}+(\vec{p} \cdot \vec{C}) \vec{C}+\vec{q} \times(\vec{p} \times \vec{q})-a \vec{C} \times \vec{q}\right) .
\end{aligned}
$$

\section{Hierarchy generated by Casimir functions of $\mathfrak{s o}_{\epsilon}(n)$}

In this section we consider some deformation the Lie algebra $\mathfrak{s o}(n)$ and the hierarchy generated by the Casimir functions for this deformation.

\subsection{The Deformed Lie Algebra}

Consider the deformation of the Lie algebra $\mathfrak{s o}(n)$ given by

$$
\left(\begin{array}{ccc}
0 & \epsilon a & \epsilon \vec{p} \\
-a & 0 & \vec{q} \\
-\vec{p}^{\top} & -\vec{q}^{\top} & C
\end{array}\right) \in \mathfrak{s o}_{\epsilon}(n), \quad \epsilon, a \in \mathbb{R}, \quad \vec{p}, \vec{q} \in \mathbb{R}^{n-2}, \quad C \in \mathfrak{s o}(n-2) .
$$

Since

$$
\begin{aligned}
& {\left[\left(\begin{array}{ccc}
0 & \epsilon a_{1} & \epsilon \vec{p}_{1} \\
-a_{1} & 0 & \vec{q}_{1} \\
-\vec{p}_{1}^{\top} & -\vec{q}_{1}^{\top} & C_{1}
\end{array}\right),\left(\begin{array}{ccc}
0 & \epsilon a_{2} & \epsilon \vec{p}_{2} \\
-a_{2} & 0 & \vec{q}_{2} \\
-\vec{p}_{2}^{\top} & -\vec{q}_{2}^{\top} & C_{2}
\end{array}\right)\right]} \\
& =\left(\begin{array}{ccc}
-\left(\vec{p}_{2} \cdot \vec{q}_{1}-\vec{p}_{1} \cdot \vec{q}_{2}\right) & \epsilon\left(\vec{p}_{2} \cdot \vec{q}_{1}-\vec{p}_{1} \cdot \vec{q}_{2}\right) & \epsilon\left(a_{1} \vec{q}_{2}-a_{2} \vec{q}_{1}+\vec{p}_{1} C_{2}-\vec{p}_{2} C_{1}\right) \\
-\left(a_{1} \vec{q}_{2}-a_{2} \vec{q}_{1}\right. & 0 & \epsilon\left(a_{2} \vec{p}_{1}-a_{1} \vec{p}_{2}\right)+\vec{q}_{1} C_{2}-\vec{q}_{2} C_{1} \\
\left.+\vec{p}_{1} C_{2}-\vec{p}_{2} C_{1}\right)^{\top} & -\left(\epsilon\left(a_{2} \vec{p}_{1}-a_{1} \vec{p}_{2}\right)\right. & \epsilon\left(\vec{p}_{2}^{\top} \vec{p}_{1}-\vec{p}_{1}^{\top} \vec{p}_{1}\right)+\vec{q}_{2}^{\top} \vec{q}_{1} \\
\left.+\vec{q}_{1} C_{2}-\vec{q}_{2} C_{1}\right)^{\top} & -\vec{q}_{1}^{\top} \vec{q}_{1}+\left[C_{1}, C_{2}\right]
\end{array}\right)
\end{aligned}
$$

it follows that the set of elements of the form (3.1) is a Lie algebra relative to the standard matrix commutator. This was proved in [13] in a more general situation. The space $\mathcal{L}_{+}$is also dual to the Lie algebra $\mathfrak{s o}_{\epsilon}(n)$ by the pairing given by the trace of the product of matrices (2.1). Thus $C^{\infty}\left(\mathcal{L}_{+}\right)$is endowed with the Lie-Poisson bracket

$$
\{f, g\}_{1, \epsilon}=\operatorname{Tr}\left(\rho\left[\frac{\partial f}{\partial \rho}, \frac{\partial g}{\partial \rho}\right]\right),
$$


where

$$
\frac{\partial f}{\partial \rho}=\left(\begin{array}{ccc}
0 & \epsilon \frac{\partial f}{\partial a} & \epsilon \frac{\partial f}{\partial \vec{p}} \\
-\frac{\partial f}{\partial a} & 0 & \frac{\partial f}{\partial \vec{q}} \\
-\frac{\partial f}{\partial \vec{p}^{\top}} & -\frac{\partial f}{\partial \vec{q}^{\top}} & \frac{\partial f}{\partial C}
\end{array}\right) .
$$

It easy to see that if $\epsilon=1$ then the Poisson bracket (3.2) coincides with (2.4). If $\epsilon=0$, the Poisson bracket (3.2) coincides with the Lie-Poisson bracket on the dual of the Euclidean algebra $\mathfrak{s e}(n-1)$. Considering the second compatible Poisson bracket associated to (3.2) for the constant matrix $\rho_{0}$ given in (2.6), we obtain the Poisson bracket (2.9).

If $\rho \in \mathcal{L}_{+}$, define

$$
\widetilde{\rho}_{\epsilon}:=\left(\begin{array}{ccc}
0 & a & \vec{p} \\
-\frac{1}{\epsilon} a & 0 & \vec{q} \\
-\frac{1}{\epsilon} \vec{p}^{\top} & -\vec{q}^{\top} & C
\end{array}\right) \in \mathfrak{s o}_{\epsilon}(n) .
$$

It is easy to see that the elements of the form $\widetilde{\rho}_{\epsilon}$ are characterized among the matrices in $\mathfrak{s l}(n, \mathbb{R})$ by the condition

$$
\widetilde{\rho}_{\epsilon} \eta+\eta \widetilde{\rho}_{\epsilon}^{\top}=0
$$

where

$$
\eta:=\left(\begin{array}{ccc}
\epsilon & 0 & \overrightarrow{0} \\
0 & 1 & \overrightarrow{0} \\
\overrightarrow{0}^{\top} & \overrightarrow{0}^{\top} & \mathbf{1}
\end{array}\right)
$$

3.2 The First Family of Functions in Involution

Let us show that the Casimir functions of (3.2) (see [13])

$$
H_{k, \epsilon}=\epsilon^{k} \operatorname{Tr} \widetilde{\rho}_{\epsilon}^{2 k}=\epsilon^{k} \operatorname{Tr}\left(\begin{array}{ccc}
0 & a & \vec{p} \\
-\frac{1}{\epsilon} a & 0 & \vec{q} \\
-\frac{1}{\epsilon} \vec{p}^{\top} & -\vec{q}^{\top} & C
\end{array}\right)^{2 k}, \quad k \in \mathbb{N},
$$

are in involution with respect to the Poisson bracket (2.9). We begin by noticing that if $\widetilde{\rho}_{\epsilon} \in \mathfrak{s o}_{\epsilon}(n)$ then $\widetilde{\rho}_{\epsilon}^{2 k-1} \in \mathfrak{s o}_{\epsilon}(n)$. Indeed, since

$$
\widetilde{\rho}_{\epsilon}=-\eta \widetilde{\rho}_{\epsilon}^{\top} \eta^{-1}
$$

we get

$$
\widetilde{\rho}_{\epsilon}^{2 k-1}=-\eta\left(\widetilde{\rho}_{\epsilon}^{2 k-1}\right)^{\top} \eta^{-1}
$$

which means that $\widetilde{\rho}_{\epsilon}^{2 k-1} \in \mathfrak{s o}_{\epsilon}(n)$. The even powers of $\widetilde{\rho}_{\epsilon}$ are deformed symmetric matrices, i.e., they satisfy the conditions

$$
\widetilde{\rho}_{\epsilon}^{2 k} \eta-\eta\left(\widetilde{\rho}_{\epsilon}^{2 k}\right)^{\top}=0
$$

as an easy verification shows.

Now we prove that $\left\{H_{k, \epsilon}, H_{l, \epsilon}\right\}_{2}=0$. Since

$$
\frac{\partial H_{k, \epsilon}}{\partial B}=-4 k \epsilon^{k}\left(\begin{array}{cc}
\frac{1}{\epsilon} & 0 \\
0 & 1
\end{array}\right) P_{+} \widetilde{\rho}_{\epsilon}^{2 k-1} P_{-},
$$




$$
\frac{\partial H_{k, \epsilon}}{\partial B^{\top}}=4 k \epsilon^{k} P_{-} \widetilde{\rho}_{\epsilon}^{2 k-1} P_{+},
$$

substitution in the Poisson bracket (2.9) yields

$$
\begin{aligned}
& \left\{H_{k, \epsilon}, H_{l, \epsilon}\right\}_{2}=\operatorname{Tr}\left(\frac{\partial H_{k, \epsilon}}{\partial B^{\top}} A_{0} \frac{\partial H_{l, \epsilon}}{\partial B}\right) \\
& =-16 k l \epsilon^{k+l} \operatorname{Tr}\left(P_{-} \widetilde{\rho}_{\epsilon}^{2 k-1} P_{+} A_{0}\left(\begin{array}{cc}
\frac{1}{\epsilon} & 0 \\
0 & 1
\end{array}\right) P_{+} \widetilde{\rho}_{\epsilon}^{2 l-1} P_{-}\right) \\
& =-16 k l \epsilon^{k+l} \operatorname{Tr}\left(\widetilde{\rho}_{\epsilon}^{2 k-1} P_{+}\left(\begin{array}{cc}
0 & 1 \\
-\frac{1}{\epsilon} & 0
\end{array}\right) P_{+} \widetilde{\rho}_{\epsilon}^{2 l-1}\right) \\
& +16 k l \operatorname{Tr}\left(P_{+} \widetilde{\rho}_{\epsilon}^{2 k-1} P_{+}\left(\begin{array}{cc}
0 & 1 \\
-\frac{1}{\epsilon} & 0
\end{array}\right) P_{+} \widetilde{\rho}_{\epsilon}^{2 l-1} P_{+}\right) \\
& =-16 k l \epsilon^{k+l}\left(\operatorname{Tr}\left(\begin{array}{cc}
0 & 1 \\
-\frac{1}{\epsilon} & 0
\end{array}\right) P_{+} \widetilde{\rho}_{\epsilon}^{2 l+2 k-2} P_{+}\right) \\
& +16 k l \operatorname{Tr}\left(P_{+} \widetilde{\rho}_{\epsilon}^{2 k-1} P_{+}\left(\begin{array}{cc}
0 & 1 \\
-\frac{1}{\epsilon} & 0
\end{array}\right) P_{+} \widetilde{\rho}_{\epsilon}^{2 l-1} P_{+}\right)=0 .
\end{aligned}
$$

The first summand vanishes because it is the product of two $2 \times 2$ matrices, one of them a deformed antisymmetric matrix and the second a deformed symmetric matrix

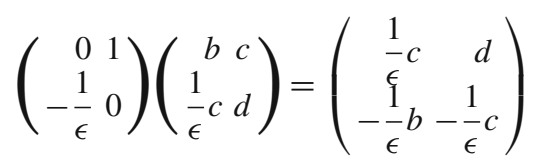

and hence the trace vanishes. The second summand vanishes because it is the product of three deformed antisymmetric $2 \times 2$ matrices.

\subsection{Involutivity of the Full Set of Functions}

In addition to the involutive family of integrals $H_{k, \epsilon}$ we have the involutive family of integrals $\delta_{k}$ given by (2.22), as we have seen in $\S 2$. Next, we prove that $\delta_{1}$ commutes with $H_{l, \epsilon}$. Indeed,

$$
\begin{aligned}
\left\{\delta_{1}, H_{l, \epsilon}\right\}_{2} & =\operatorname{Tr}\left(\frac{\partial \delta_{1}}{\partial B^{\top}} A_{0} \frac{\partial H_{l, \epsilon}}{\partial B}\right)=-8 l \epsilon^{l} \operatorname{Tr}\left(C B^{\top} A_{0}^{2}\left(\begin{array}{cc}
\frac{1}{\epsilon} & 0 \\
0 & 1
\end{array}\right) P_{+} \widetilde{\rho}_{\epsilon}^{2 l-1} P_{-}\right) \\
& =-8 l \epsilon^{l} \operatorname{Tr}\left(P_{-} \widetilde{\rho}_{\epsilon} P_{-} \widetilde{\rho}_{\epsilon} P_{+} \widetilde{\rho}_{\epsilon}^{2 l-1} P_{-}\right) \\
& =-8 l \epsilon^{l} \operatorname{Tr}\left(\widetilde{\rho}_{\epsilon} P_{-} \widetilde{\rho}_{\epsilon} P_{+} \widetilde{\rho}_{\epsilon}^{2 l-1}\right)+8 l \epsilon^{l} \operatorname{Tr}\left(P_{+} \widetilde{\rho}_{\epsilon} P_{-} \widetilde{\rho}_{\epsilon} P_{+} \widetilde{\rho}_{\epsilon}^{2 l-1} P_{+}\right) \\
& =-8 l \epsilon^{l}\left[\operatorname{Tr}\left(\widetilde{\rho}_{\epsilon} P_{+} \widetilde{\rho}_{\epsilon}^{2 l}\right)-\operatorname{Tr}\left(P_{+} \widetilde{\rho}_{\epsilon} P_{+} \widetilde{\rho}_{\epsilon}^{2 l} P_{+}\right)-\operatorname{Tr}\left(P_{-} \widetilde{\rho}_{\epsilon} P_{+} \widetilde{\rho}_{\epsilon}^{2 l-1} P_{+} \widetilde{\rho}_{\epsilon} P_{-}\right)\right] \\
& =-8 l \epsilon^{l} \operatorname{Tr}\left(\widetilde{\rho}_{\epsilon} P_{+} \widetilde{\rho}_{\epsilon}^{2 l}\right)=-8 l \epsilon^{l} \operatorname{Tr}\left(P_{+} \widetilde{\rho}_{\epsilon}^{2 l+1} P_{+}\right)=0 .
\end{aligned}
$$

In the proof we used the following properties:

1. in the third equality we used the identity

$$
B^{\top}\left(\begin{array}{cc}
\frac{1}{\epsilon} & 0 \\
0 & 1
\end{array}\right)=-P_{-} \tilde{\rho}_{\epsilon} P_{+} ;
$$


2. the matrix $P_{-} \widetilde{\rho}_{\epsilon} P_{+} \widetilde{\rho}_{\epsilon}^{2 l-1} P_{+} \widetilde{\rho}_{\epsilon} P_{-}$is antisymmetric because it is of the form

$$
\left(\frac{1}{\epsilon} \vec{p}^{\top} \vec{q}^{\top}\right)\left(\begin{array}{cc}
0 & c \\
-\frac{1}{\epsilon} c & 0
\end{array}\right)\left(\begin{array}{l}
\vec{p} \\
\vec{q}
\end{array}\right)=-\frac{1}{\epsilon} c\left(\vec{q}^{\top} \vec{p}-\vec{p}^{\top} \vec{q}\right) ;
$$

3. the matrix $P_{+} \widetilde{\rho}_{\epsilon} P_{+} \widetilde{\rho}_{\epsilon}^{2 l} P_{+}$is a product of a deformed antisymmetric and a deformed symmetric matrix like in (3.13) which has zero trace.

Next, we show that $\left\{\delta_{k}, H_{l, \epsilon}\right\}_{2}=0$. Indeed,

$$
\begin{aligned}
& \left\{\delta_{k}, H_{l, \epsilon}\right\}_{2}=\operatorname{Tr}\left(\frac{\partial \delta_{k}}{\partial B^{\top}} A_{0} \frac{\partial H_{l, \epsilon}}{\partial B}\right) \\
& =-8 l \epsilon^{l} \operatorname{Tr}\left(C^{2 k-1} B^{\top} A_{0}^{2}\left(\begin{array}{cc}
\frac{1}{\epsilon} & 0 \\
0 & 1
\end{array}\right) P_{+} \widetilde{\rho}_{\epsilon}^{2 l-1} P_{-}\right) \\
& \stackrel{(3.15)}{=}-8 l \epsilon^{l} \operatorname{Tr}\left(\left(P_{-} \widetilde{\rho}_{\epsilon} P_{-}\right)^{2 k-1} P_{-} \widetilde{\rho}_{\epsilon} P_{+} \widetilde{\rho}_{\epsilon}^{2 l-1} P_{-}\right) \\
& =-8 l \epsilon^{l} \operatorname{Tr}\left(P_{-} \tilde{\rho}_{\epsilon} P_{-}\left(P_{-} \tilde{\rho}_{\epsilon} P_{-}\right)^{2 k-2} P_{-} \tilde{\rho}_{\epsilon} P_{+} \widetilde{\rho}_{\epsilon}^{2 l-1} P_{-}\right) \\
& =-8 l \epsilon^{l} \operatorname{Tr}\left(\tilde{\rho}_{\epsilon}\left(P_{-} \tilde{\rho}_{\epsilon} P_{-}\right)^{2 k-2} P_{-} \tilde{\rho}_{\epsilon} P_{+} \widetilde{\rho}_{\epsilon}^{2 l-1}\right) \\
& +8 l \epsilon^{l} \operatorname{Tr}\left(P_{+} \widetilde{\rho}_{\epsilon} P_{-}\left(P_{-} \widetilde{\rho}_{\epsilon} P_{-}\right)^{2 k-2} P_{-} \widetilde{\rho}_{\epsilon} P_{+} \widetilde{\rho}_{\epsilon}^{2 l-1} P_{+}\right) \\
& =-8 l \epsilon^{l} \operatorname{Tr}\left(\left(P_{-} \widetilde{\rho}_{\epsilon} P_{-}\right)^{2 k-2} P_{-} \widetilde{\rho}_{\epsilon} P_{+} \widetilde{\rho}_{\epsilon}^{2 l}\right) \\
& +8 l \epsilon^{l} \operatorname{Tr}\left(\left(P_{-} \widetilde{\rho}_{\epsilon} P_{+}\right)\left(P_{+} \widetilde{\rho}_{\epsilon}^{2 l-1} P_{+}\right)\left(P_{+} \widetilde{\rho}_{\epsilon} P_{-}\right)\left(P_{-} \widetilde{\rho}_{\epsilon} P_{-}\right)^{2 k-2}\right) \\
& =-8 l \epsilon^{l} \operatorname{Tr}\left(P_{-} \widetilde{\rho}_{\epsilon}\left(P_{-} \widetilde{\rho}_{\epsilon} P_{-}\right)^{2 k-3} \widetilde{\rho}_{\epsilon} P_{+} \widetilde{\rho}_{\epsilon}^{2 l}\right) \\
& =-8 l \epsilon^{l} \operatorname{Tr}\left(\widetilde{\rho}_{\epsilon}\left(P_{-} \widetilde{\rho}_{\epsilon} P_{-}\right)^{2 k-3} P_{-} \tilde{\rho}_{\epsilon} P_{+} \widetilde{\rho}_{\epsilon}^{2 l}\right) \\
& +8 l \epsilon^{l} \operatorname{Tr}\left(\left(P_{+} \widetilde{\rho}_{\epsilon} P_{-}\left(P_{-} \tilde{\rho}_{\epsilon} P_{-}\right)^{2 k-3} P_{-} \widetilde{\rho}_{\epsilon} P_{+}\right) P_{+} \widetilde{\rho}_{\epsilon}^{2 l} P_{+}\right) \\
& \stackrel{(3.15)}{=}-8 l \epsilon^{l} \operatorname{Tr}\left(\left(P_{-} \widetilde{\rho}_{\epsilon} P_{-}\right)^{2(k-1)-1} P_{-} \widetilde{\rho}_{\epsilon} P_{+} \widetilde{\rho}_{\epsilon}^{2(l+1)-1}\right) \\
& =-8 l \epsilon^{l} \operatorname{Tr}\left(C^{2(k-1)-1} B^{\top} A_{0}^{2}\left(\begin{array}{cc}
\frac{1}{\epsilon} & 0 \\
0 & 1
\end{array}\right) P_{+} \widetilde{\rho}_{\epsilon}^{2(l+1)-1} P_{-}\right) \\
& =\frac{l}{(l+1) \epsilon}\left\{\delta_{k-1}, H_{l+1, \epsilon}\right\}_{2} \text {. }
\end{aligned}
$$

In the seventh equality we use $\operatorname{Tr}\left(\left(P_{-} \widetilde{\rho}_{\epsilon} P_{+}\right)\left(P_{+} \widetilde{\rho}_{\epsilon}^{2 l-1} P_{+}\right)\left(P_{+} \widetilde{\rho}_{\epsilon} P_{-}\right)\left(P_{-} \widetilde{\rho}_{\epsilon} P_{-}\right)^{2 k-2}\right)=0$, because the matrix $\left(P_{-} \widetilde{\rho}_{\epsilon} P_{+}\right)\left(P_{+} \widetilde{\rho}_{\epsilon}^{2 l-1} P_{+}\right)\left(P_{+} \widetilde{\rho}_{\epsilon} P_{-}\right)$is antisymmetric (like in (3.16)) and the matrix $\left(P_{-} \widetilde{\rho}_{\epsilon} P_{-}\right)^{2 k-2}=C^{2 k-2}$ is symmetric. In the ninth equality, we use the vanishing of the trace of the matrix $\left(P_{+} \widetilde{\rho}_{\epsilon} P_{-}\left(P_{-} \widetilde{\rho}_{\epsilon} P_{-}\right)^{2 k-3} P_{-} \widetilde{\rho}_{\epsilon} P_{+}\right) P_{+} \widetilde{\rho}_{\epsilon}^{2 l} P_{+}$. Indeed,

$$
\begin{aligned}
-P_{+} \tilde{\rho}_{\epsilon} P_{-}\left(P_{-} \tilde{\rho}_{\epsilon} P_{-}\right)^{2 k-3} P_{-} \tilde{\rho}_{\epsilon} P_{+} & =\left(\begin{array}{c}
\vec{p} \\
\vec{q}
\end{array}\right) C^{2 k-3}\left(\frac{1}{\epsilon} \vec{p}^{\top} \vec{q}^{\top}\right) \\
& =\left(\begin{array}{c}
\frac{1}{\epsilon} \vec{p} C^{2 k-3} \vec{p}^{\top} \vec{p} C^{2 k-3} \vec{q}^{\top} \\
\frac{1}{\epsilon} \vec{q} C^{2 k-3} \vec{p}^{\top} \vec{q} C^{2 k-3} \vec{q}^{\top}
\end{array}\right)
\end{aligned}
$$

is deformed antisymmetric and $P_{+} \widetilde{\rho}_{\epsilon}^{2 l} P_{+}$is deformed symmetric, like in (3.13).

Equations (3.14) and (3.17) show that $\left\{\delta_{k}, H_{l, \epsilon}\right\}_{2}=0$ for all $k, l \in \mathbb{N}$. Thus the family of functions $\left\{H_{l, \epsilon}, \delta_{k} \mid k, l \in \mathbb{N}\right\}$ are in involution. 
The candidates for the independent integrals depend on whether $n$ is even or odd. If $n=2 p+1$, we take $H_{1, \epsilon}, \ldots, H_{p, \epsilon}, \delta_{1}, \ldots, \delta_{p-1}$ as the system of integrals in involution. Hence we have $2 p-1=n-2$ integrals in involution. If $n=2 p$, we take $H_{1, \epsilon}, \ldots, H_{p-1, \epsilon}, \delta_{1}, \ldots, \delta_{p-1}$ as the system of integrals in involution. Hence we have $2 p-2=n-2$ integrals in involution.

Example 3.1 For $k=1,2$ the Hamiltonians $H_{k, \epsilon}$ are

$$
\begin{aligned}
& H_{1, \epsilon}=-2\left(a^{2}+\|\vec{p}\|^{2}+\epsilon\|\vec{q}\|^{2}\right)+\epsilon \operatorname{Tr} C^{2} \\
& \begin{aligned}
H_{2, \epsilon}=2 a^{4}+\epsilon^{2} \operatorname{Tr} C^{4}+2\|\vec{p}\|^{4}+2 \epsilon^{2}\|\vec{q}\|^{4}+4 a^{2}\|\vec{p}\|^{2}+4 \epsilon a^{2}\|\vec{q}\|^{2} \\
\quad+4 \epsilon(\vec{p} \cdot \vec{q})^{2}+8 \epsilon a \vec{p} C \cdot \vec{q}-4 \epsilon^{2} \vec{q} C^{2} \cdot \vec{q}-4 \epsilon \vec{p} C^{2} \cdot \vec{p} .
\end{aligned}
\end{aligned}
$$

Let us consider the case when $\widetilde{\rho}_{\epsilon}$ is $5 \times 5$ matrix, so $\vec{p}, \vec{q} \in \mathbb{R}^{3}$,

$$
C=\left(\begin{array}{ccc}
0 & -c_{3} & c_{2} \\
c_{3} & 0 & -c_{1} \\
-c_{2} & c_{1} & 0
\end{array}\right) \in \mathfrak{s o}(3),
$$

and define $\vec{C}:=\left(c_{1}, c_{2}, c_{3}\right)$. Thus, $\operatorname{Tr} C^{2}=-2\|\vec{C}\|^{2}, \operatorname{Tr} C^{4}=2\|\vec{C}\|^{4}, \vec{q} C=\vec{q} \times \vec{C}$, and $\vec{q} C^{2}=(\vec{C} \cdot \vec{q}) \vec{C}-\|\vec{C}\|^{2} \vec{q}$.

The integrals (3.19) and (3.20) have thus the form

$$
\begin{aligned}
& H_{1, \epsilon}=-2\left(a^{2}+\epsilon\|\vec{C}\|^{2}+\|\vec{p}\|^{2}+\epsilon\|\vec{q}\|^{2}\right), \\
& H_{2, \epsilon}=\frac{1}{2} H_{1, \epsilon}^{2}-4 \epsilon\left(\epsilon(\vec{q} \cdot \vec{C})^{2}+(\vec{p} \cdot \vec{C})^{2}+\|-a \vec{C}+\vec{q} \times \vec{p}\|^{2}\right),
\end{aligned}
$$

and we have an additional integral in involution

$$
\delta_{1}=-2 \vec{C} \cdot(\vec{q} \times \vec{p}) .
$$

Combining the Hamiltonians $H_{1, \epsilon}, H_{2, \epsilon}$ with $\delta_{1}$, we find that the three following functions are also in involution

$$
\begin{aligned}
& h_{1, \epsilon}=\frac{1}{2}\left(\|\vec{p}\|^{2}+\epsilon\|\vec{q}\|^{2}\right), \\
& h_{2, \epsilon}=\frac{1}{2} \epsilon(\vec{q} \cdot \vec{C})^{2}+\frac{1}{2}(\vec{p} \cdot \vec{C})^{2}+\frac{1}{2}\|\vec{q} \times \vec{p}\|^{2}
\end{aligned}
$$

and $\delta_{1}$. Hamilton's equations for $h_{2, \epsilon}$ are

$$
\begin{aligned}
& \frac{\partial \vec{p}}{\partial t}=\epsilon(\vec{q} \cdot \vec{C}) \vec{C}-\vec{p} \times(\vec{p} \times \vec{q}), \\
& \frac{\partial \vec{q}}{\partial t}=-(\vec{p} \cdot \vec{C}) \vec{C}-\vec{q} \times(\vec{p} \times \vec{q}) .
\end{aligned}
$$

If we let $\epsilon=0$ then the functions

$$
\begin{aligned}
& h_{1,0}=\frac{1}{2}\|\vec{p}\|^{2}, \\
& h_{2,0}=\frac{1}{2}(\vec{p} \cdot \vec{C})^{2}+\frac{1}{2}\|\vec{q} \times \vec{p}\|^{2}
\end{aligned}
$$


and $\delta_{1}$ are also in involution. Hamilton's equations for $h_{2,0}$ are

$$
\begin{aligned}
& \frac{\partial \vec{p}}{\partial t}=-\vec{p} \times(\vec{p} \times \vec{q})=\|\vec{p}\|^{2} \vec{q}-(\vec{p} \cdot \vec{q}) \vec{p} \\
& \frac{\partial \vec{q}}{\partial t}=-(\vec{p} \cdot \vec{C}) \vec{C}-\vec{q} \times(\vec{p} \times \vec{q})=-(\vec{p} \cdot \vec{C}) \vec{C}+(\vec{p} \cdot \vec{q}) \vec{q}-\|\vec{q}\|^{2} \vec{p}
\end{aligned}
$$

The Hamiltonian $h_{2,0}$ has the symmetry $\vec{q} \mapsto \vec{q}+\lambda \vec{p}$ and $\vec{p} \mapsto \vec{p}$ whose momentum map is $h_{1,0}$ We can perform a Hamiltonian reduction at the value $h_{1,0}=1 / 2$. The reduced equations of motion are equivalent to the equation of motion for the classical Neumann system. Indeed, the phase space of the reduced system is $T S^{2}$, i.e., it is given by

$$
\left\{(\mathbf{x}, \mathbf{y}) \in \mathbb{R}^{3} \times \mathbb{R}^{3} \mid\|\mathbf{x}\|=1, \mathbf{x} \cdot \mathbf{y}=0\right\}
$$

because the map $h_{1,0}^{-1}(1 / 2) \ni(\vec{p}, \vec{q}) \mapsto(\mathbf{x}, \mathbf{y}):=\left((\vec{p}, \vec{q}-(\vec{p} \cdot \vec{q}) \vec{p}) \in T S^{2}\right.$ descends to a symplectic diffeomorphism of the reduced phase space $h_{1,0}^{-1}(1 / 2) / \mathbb{R}$ onto $T S^{2}$. A direct verification shows that the reduced equations of motion induced by $h_{2,0}$ are

$$
\begin{aligned}
& \frac{\partial \mathbf{x}}{\partial t}=\mathbf{y} \\
& \frac{\partial \mathbf{y}}{\partial t}=-(\mathbf{x} \cdot \vec{C}) \vec{C}-\left(\|\mathbf{y}\|^{2}-(\mathbf{x} \cdot \vec{C})^{2}\right) \mathbf{x}
\end{aligned}
$$

i.e., the classical Neumann system equations describing the motion of a particle on $S^{2}$ under the influence of the quadratic potential $\frac{1}{2} \mathbf{x}\left(C^{2}+\|\vec{C}\|^{2} \mathbf{1}\right) \cdot \mathbf{x}$ ([12]; for the $N$-dimensional generalization see [15]).

We have three functions $h_{1, \epsilon}, h_{2, \epsilon}, \delta_{1}$ and we calculate

$$
\begin{aligned}
& \frac{\partial h_{1, \epsilon}}{\partial \vec{p}^{\top}}=\vec{p}^{\top}, \\
& \frac{\partial h_{2, \epsilon}}{\partial \vec{p}^{\top}}=(\vec{p} \cdot \vec{C}) \vec{C}^{\top}+\vec{q}^{\top} \times\left(\vec{p}^{\top} \times \vec{q}^{\top}\right)=(\vec{p} \cdot \vec{C}) \vec{C}^{\top}+\|\vec{q}\|^{2} \vec{p}^{\top}-(\vec{p} \cdot \vec{q}) \vec{q}^{\top}, \\
& \frac{\partial \delta_{1}}{\partial \vec{p}^{\top}}=-2 \vec{C}^{\top} \times \vec{q}^{\top} .
\end{aligned}
$$

The Jacobian of these functions is given by

$$
\begin{aligned}
J & =\left|\frac{\partial\left(h_{1, \epsilon}, h_{2, \epsilon}, \delta_{1}\right)}{\partial \vec{p}^{\top}}\right| \\
& =2\left((\vec{p} \cdot \vec{C}) \vec{C}^{\top}-(\vec{p} \cdot \vec{q}) \vec{q}^{\top}\right) \cdot\left(\vec{p}^{\top} \times\left(\vec{C}^{\top} \times \vec{q}^{\top}\right)\right) \\
& =2\left((\vec{p} \cdot \vec{C}) \vec{C}^{\top}-(\vec{p} \cdot \vec{q}) \vec{q}^{\top}\right) \cdot\left((\vec{p} \cdot \vec{q}) \vec{C}^{\top}-(\vec{p} \cdot \vec{C}) \vec{q}^{\top}\right) .
\end{aligned}
$$

Setting the expression in (3.38) equal to zero yields a hypersurface in $\mathbb{R}^{6}$ and hence on its complement, which is a Zariski open set hence dense in $\mathbb{R}^{6}$, the Jacobian does not vanish. This proves the independence of the functions $h_{1, \epsilon}, h_{2, \epsilon}, \delta_{1}$. 
Example 3.2 In this example we consider the case when $\widetilde{\rho}_{\epsilon}$ is $6 \times 6$-matrix then the Hamiltonians exactly have the form

$$
\begin{aligned}
H_{1, \epsilon}= & -2\left(a^{2}+\|\vec{p}\|^{2}+\epsilon\|\vec{q}\|^{2}+\epsilon\left(c_{1}^{2}+c_{2}^{2}+c_{3}^{2}+c_{4}^{2}+c_{5}^{2}+c_{6}^{2}\right)\right), \\
H_{2, \epsilon}= & \frac{1}{2} H_{1, \epsilon}^{2}-4 \epsilon\left(\epsilon\left(c_{1}^{2}+c_{2}^{2}+c_{3}^{2}+c_{4}^{2}+c_{5}^{2}+c_{6}^{2}\right)\|\vec{q}\|^{2}+\epsilon \vec{q} C^{2} \vec{q}^{\top}+\frac{1}{2} \sum_{k \neq l} J_{k l}^{2}+\right. \\
& \left.+\left(c_{1}^{2}+c_{2}^{2}+c_{3}^{2}+c_{4}^{2}+c_{5}^{2}+c_{6}^{2}\right)\|\vec{p}\|^{2}+\vec{p} C^{2} \vec{p}^{\top}+\epsilon\left(c_{1} c_{6}+c_{3} c_{4}-c_{2} c_{5}\right)^{2}\right),
\end{aligned}
$$

and we have additional integrals in involution

$$
\begin{aligned}
\delta_{1}=2 \vec{q} C \vec{p}^{\top}, & \\
\delta_{2}=2 \vec{q} C^{3} \vec{p}^{\top}= & -2\left(c_{1}^{2}+c_{2}^{2}+c_{3}^{2}+c_{4}^{2}+c_{5}^{2}+c_{6}^{2}\right) \delta_{1} \\
& +2\left(c_{1} c_{6}+c_{3} c_{4}-c_{2} c_{5}\right) \vec{q}\left(\begin{array}{rrrr}
0 & c_{6} & -c_{5} & c_{4} \\
-c_{6} & 0 & c_{3} & -c_{2} \\
c_{5} & -c_{3} & 0 & c_{1} \\
-c_{4} & c_{2} & -c_{1} & 0
\end{array}\right) \vec{p}^{\top},
\end{aligned}
$$

where

$$
\begin{aligned}
C & :=\left(\begin{array}{cccc}
0 & c_{1} & c_{2} & c_{3} \\
-c_{1} & 0 & c_{4} & c_{5} \\
-c_{2} & -c_{4} & 0 & c_{6} \\
-c_{3} & -c_{5} & -c_{6} & 0
\end{array}\right), \\
J_{k l} & :=a C_{k l}+p_{l} q_{k}-p_{k} q_{l},
\end{aligned}
$$

and $C_{k l}$ is $(k, l)$ entry of the matrix $C$. Hamilton's equations for $H_{2, \epsilon}$ are again connected to the Neumann model.

Note that

$$
\begin{aligned}
H_{3, \epsilon}= & \left(\frac{1}{4}-\frac{3}{8} \epsilon\right) H_{1, \epsilon}^{3}+\frac{3}{4} \epsilon H_{2, \epsilon} H_{1, \epsilon} \\
& -\epsilon^{2}\left[a\left(c_{1} c_{6}+c_{3} c_{4}-c_{2} c_{5}\right)+\frac{1}{2}\left(\delta_{3}+\left(c_{1}^{2}+c_{2}^{2}+c_{3}^{2}+c_{4}^{2}+c_{5}^{2}+c_{6}^{2}\right) \delta_{1}\right)\right]^{2} .
\end{aligned}
$$

\section{Independence of integrals}

4.1 Independence of the Integrals $\delta_{1}, \ldots, \delta_{p-1}$

Let $n=2 p+1$ or $n=2 p$. We shall prove that the functions $\delta_{1}, \ldots, \delta_{p-1}$ are independent. Note that $\delta_{k}$ given by formula (2.22) can be rewritten in the form

$$
\delta_{k}(\vec{p}, \vec{q})=-2 \vec{p} C^{2 k-1} \vec{q}^{\top} .
$$

From the above we obtain

$$
\frac{\partial \delta_{k}}{\partial \vec{p}}=2 \vec{q} C^{2 k-1}
$$


The $(n-2) \times(n-2)$ matrix $C$ is antisymmetric, so it can be presented in the form

$$
\begin{aligned}
& C=Q^{\top} D Q=Q^{\top}\left(\begin{array}{rr}
0 & \Lambda \\
-\Lambda & 0
\end{array}\right) Q \quad \text { if } n=2 p, \\
& C=Q^{\top} D Q=Q^{\top}\left(\begin{array}{rcc}
0 & \Lambda & 0 \\
-\Lambda & 0 & 0 \\
0 & 0 & 0
\end{array}\right) Q \text { if } n=2 p+1,
\end{aligned}
$$

where $Q \in S O(n-2)$ and $\Lambda=\operatorname{diag}\left(\lambda_{1}, \ldots, \lambda_{p-1}\right)$. Therefore we have

$$
\frac{\partial \delta_{k}}{\partial \vec{p}}=2 \vec{q} Q^{\top} D^{2 k-1} Q=2 \mathbf{v} D^{2 k-1} Q,
$$

where $\mathbf{v}:=\vec{q} Q^{\top}$. We consider the generic case $0<\lambda_{1}<\lambda_{2}<\cdots<\lambda_{p-1}$. Now we check the linear independence of $\delta_{1}, \ldots, \delta_{p-1}$. Suppose

$$
\left(\alpha_{1} \mathbf{v}+\alpha_{2} \mathbf{v} D^{2}+\cdots+\alpha_{p-1} \mathbf{v} D^{2 p-4}\right) D Q=0
$$

for $\alpha_{1}, \ldots, \alpha_{p-1} \in \mathbb{R}$. Because

$$
\begin{aligned}
D^{2 m} & =(-1)^{m}\left(\begin{array}{cc}
\Lambda^{2 m} & 0 \\
0 & \Lambda^{2 m}
\end{array}\right) \quad \text { if } n=2 p, \\
D^{2 m} & =(-1)^{m}\left(\begin{array}{ccc}
\Lambda^{2 m} & 0 & 0 \\
0 & \Lambda^{2 m} & 0 \\
0 & 0 & 0
\end{array}\right) \text { if } n=2 p+1,
\end{aligned}
$$

if we denote $\mathbf{v}=(\mathbf{u}, \mathbf{w})$ or $\mathbf{v}=\left(\mathbf{u}, \mathbf{w}, v_{3}\right)$, respectively, then the equality (4.5) implies

$$
\alpha_{1} \mathbf{u}-\alpha_{2} \mathbf{u} \Lambda^{2}+\cdots+(-1)^{p} \alpha_{p-1} \mathbf{u} \Lambda^{2(p-2)}=0,
$$

or in matrix form

$$
\left(\begin{array}{cccc}
u_{1} & \lambda_{1}^{2} u_{1} & \ldots & \lambda_{1}^{2(p-2)} u_{1} \\
u_{2} & \lambda_{2}^{2} u_{2} & \ldots & \lambda_{2}^{2(p-2)} u_{2} \\
\ldots & \ldots & \ldots & \ldots \\
u_{p-1} & \lambda_{p-1}^{2} u_{p-1} & \ldots & \lambda_{p-1}^{2(p-2)} u_{p-1}
\end{array}\right)\left(\begin{array}{c}
\alpha_{1} \\
-\alpha_{2} \\
\vdots \\
(-1)^{p} \alpha_{p-1}
\end{array}\right)=0
$$

where $\mathbf{u}=\left(u_{1}, \ldots, u_{p-1}\right)$. This is a $(p-1) \times(p-1)$ linear homogeneous system with unknowns $\alpha_{1},-\alpha_{2}, \alpha_{3}, \ldots,(-1)^{p} \alpha_{p-1}$. The determinant of this system is

$$
u_{1} \cdots u_{p-1}\left|\begin{array}{cccc}
1 & \lambda_{1}^{2} & \ldots & \lambda_{1}^{2(p-2)} \\
1 & \lambda_{2}^{2} & \ldots & \lambda_{2}^{2(p-2)} \\
\ldots & \ldots & \ldots & \ldots \\
1 & \lambda_{p-1}^{2} & \ldots & \lambda_{p-1}^{2(p-2)}
\end{array}\right|=u_{1} \cdots u_{p-1} \prod_{1 \leq i<j \leq p-1}\left(\lambda_{j}^{2}-\lambda_{i}^{2}\right) \text {. }
$$

Generically, all $u_{i} \neq 0$ for $i=1, \ldots, p-1$, which implies that $\alpha_{i}=0$ for all $i=1, \ldots p-1$, thus proving that $\delta_{1}, \ldots, \delta_{p-1}$ are independent.

4.2 Independence of the Integrals $H_{1}, \ldots H_{p}$

Let $n=2 p+1$ or $n=2 p$. We shall prove that the functions $H_{1}, \ldots, H_{p}$ are independent (we will consider only the case when $\epsilon=1$; the proof when the $\epsilon \neq 1$ is similar). Since $H_{k}$ 
and $\frac{\partial H_{k}}{\partial B}$ are given by formulas (2.10) and (2.11), respectively, we get

$$
\frac{\partial H_{k}}{\partial \vec{p}}=-4 k P_{+}^{1} \widetilde{\rho}^{2 k-1} P_{-},
$$

where $P_{+}^{1}, P_{-}$are the orthogonal projectors given, in block matrix notation, by

$$
P_{+}^{1}=\left(\begin{array}{cc|c}
1 & 0 & 0 \\
0 & 0 & 0 \\
\hline 0 & 0 & \mathbf{0}
\end{array}\right), \quad P_{-}=\left(\begin{array}{lll|l}
0 & 0 & 0 \\
0 & 0 & 0 \\
\hline 0 & 0 & \mathbf{1}
\end{array}\right) .
$$

The $n \times n$ matrix $\widetilde{\rho}$ is antisymmetric, so it can be presented in the form

$$
\begin{gathered}
\tilde{\rho}=R^{\top} E R=R^{\top}\left(\begin{array}{cc|cc}
0 & \omega_{1} & 0 & 0 \\
-\omega_{1} & 0 & 0 & 0 \\
\hline 0 & 0 & 0 & \Omega \\
0 & 0 & -\Omega & 0
\end{array}\right) R \quad \text { if } n=2 p, \\
\tilde{\rho}=R^{\top} E R=R^{\top}\left(\begin{array}{rc|rrr}
0 & \omega_{1} & 0 & 0 & 0 \\
-\omega_{1} & 0 & 0 & 0 & 0 \\
\hline 0 & 0 & 0 & \Omega & 0 \\
0 & 0 & -\Omega & 0 & 0 \\
0 & 0 & 0 & 0 & 0
\end{array}\right) R \text { if } n=2 p+1,
\end{gathered}
$$

where $R \in S O(n)$ and $\Omega=\operatorname{diag}\left(\omega_{2}, \ldots, \omega_{p}\right)$. Therefore, by (4.10), we have

$$
\frac{\partial H_{k}}{\partial \vec{p}}=-4 k P_{+}^{1} R^{\top} E^{2 k-1} R P_{-} .
$$

We consider the generic case $0<\omega_{1}<\omega_{2}<\cdots<\omega_{p}$ and we write the matrix $R^{\top}$ in block form as

$$
R^{\top}=\left(\begin{array}{cc|c}
a_{1} & a_{2} & \mathbf{b}_{1} \\
a_{3} & a_{4} & \mathbf{b}_{2} \\
\hline \mathbf{d}_{1}^{\top} & \mathbf{d}_{2}^{\top} & \mathbf{G}
\end{array}\right),
$$

with $\mathbf{b}_{\mathbf{1}}=\left(b_{11}, \cdots, b_{1, n-2}\right), \mathbf{b}_{\mathbf{2}}=\left(b_{21}, \cdots, b_{2, n-2}\right), \mathbf{d}_{\mathbf{1}}=\left(d_{11}, \cdots, d_{1, n-2}\right), \mathbf{d}_{\mathbf{2}}=$ $\left(d_{21}, \cdots, d_{2, n-2}\right)$ and $\mathbf{G} \in \mathfrak{g l}(n-2, \mathbb{R})$. After a simple calculation, formula (4.13) can be rewritten in the form

$$
\begin{aligned}
\frac{\partial H_{k}}{\partial \vec{p}}= & (-1)^{k} 4 k\left(\left(-\omega_{1}^{k} a_{2}, \omega_{1}^{k} a_{1}\right)\left(\begin{array}{l}
\mathbf{d}_{\mathbf{1}} \\
\mathbf{d}_{\mathbf{2}}
\end{array}\right)\right. \\
& \left.+\left(-\omega_{2}^{k} b_{1 p},-\omega_{3}^{k} b_{1, p+1}, \ldots,-\omega_{p}^{k} b_{1,2 p-2}, \omega_{2}^{k} b_{11}, \omega_{3}^{k} b_{12}, \ldots, \omega_{p}^{k} b_{1, p-1}\right) \mathbf{G}^{\top}\right),
\end{aligned}
$$

if $n=2 p$ and in the form

$$
\begin{aligned}
\frac{\partial H_{k}}{\partial \vec{p}}= & (-1)^{k} 4 k\left(\left(-\omega_{1}^{k} a_{2}, \omega_{1}^{k} a_{1}\right)\left(\begin{array}{l}
\mathbf{d}_{\mathbf{1}} \\
\mathbf{d}_{\mathbf{2}}
\end{array}\right)\right. \\
& \left.+\left(-\omega_{2}^{k} b_{1 p},-\omega_{3}^{k} b_{1, p+1}, \ldots,-\omega_{p}^{k} b_{1,2 p-2}, \omega_{2}^{k} b_{11}, \omega_{3}^{k} b_{12}, \ldots, \omega_{p}^{k} b_{1, p-1}, 0\right) \mathbf{G}^{\top}\right),
\end{aligned}
$$


if $n=2 p+1$. Assume, generically, that $\mathbf{G}^{\top}$ has an inverse and denote

$$
\left(\begin{array}{l}
\mathbf{d}_{\mathbf{1}}^{\prime} \\
\mathbf{d}_{\mathbf{2}}^{\prime}
\end{array}\right)=\left(\begin{array}{l}
\mathbf{d}_{\mathbf{1}} \\
\mathbf{d}_{\mathbf{2}}
\end{array}\right)\left(\mathbf{G}^{\top}\right)^{-1} .
$$

Now we check the linear independence of the differentials of $H_{1}, \ldots, H_{p}$ at a generic point $\left(\begin{array}{c}\vec{p} \\ \vec{q}\end{array}\right) \in \mathbb{R}^{2(n-2)}$. Suppose

$$
\beta_{1} \frac{\partial H_{1}}{\partial \vec{p}}+\beta_{2} \frac{\partial H_{2}}{\partial \vec{p}}+\cdots+\beta_{p} \frac{\partial H_{p}}{\partial \vec{p}}=0
$$

For $n=2 p$, this is equivalent to the linear homogeneous system

$$
\begin{aligned}
& \left(\begin{array}{lll}
\omega_{1} d_{1}^{\prime \prime}-\omega_{2} b_{1 p} & \omega_{1}^{3} d_{1}^{\prime \prime}-\omega_{2}^{3} b_{1 p} & \ldots \omega_{2}^{2 p-1} d_{1}^{\prime \prime}-\omega_{2}^{2 p-1} b_{1 p} \\
\omega_{1} d_{2}^{\prime \prime}-\omega_{3} b_{1, p+1} & \omega_{1}^{3} d_{2}^{\prime \prime}-\omega_{3}^{3} b_{1, p+1} & \ldots \omega_{1}^{2 p-1} d_{2}^{\prime \prime}-\omega_{3}^{2 p-1} b_{1, p+1} \\
\ldots & \ldots & \ldots \omega^{2 p-1} \\
\omega_{1} d_{p-1}^{\prime \prime}-\omega_{p} b_{1,2 p-2} & \omega_{1}^{3} d_{p-1}^{\prime \prime}-\omega_{p}^{3} b_{1,2 p-2} \ldots \omega_{1}^{2 p-1} d_{p-1}^{\prime \prime}-\omega_{p}^{2 p-1} b_{1,2 p-2} \\
\omega_{1} d_{p}^{\prime \prime}+\omega_{2} b_{11} & \omega_{1}^{3} d_{p}^{\prime \prime}+\omega_{2}^{3} b_{11} & \ldots \omega_{1}^{2 p-1} d_{p}^{\prime \prime}+\omega_{2}^{2 p-1} b_{11} \\
\omega_{1} d_{p+1}^{\prime \prime}+\omega_{3} b_{12} & \omega_{1}^{3} d_{p+1}^{\prime \prime}+\omega_{3}^{3} b_{12} & \ldots \omega_{1}^{2 p-1} d_{p+1}^{\prime \prime}+\omega_{3}^{2 p-1} b_{12} \\
\ldots & \ldots & \ldots \omega^{2 p-1} d_{2 p-2}^{\prime \prime}+\omega_{p}^{2 p-1} b_{1, p-1}
\end{array}\right) \\
& \omega_{1} d_{2 p-2}^{\prime \prime}+\omega_{p} b_{1, p-1} \omega_{1}^{3} d_{2 p-2}^{\prime \prime}+\omega_{p}^{3} b_{1, p-1} \ldots \omega_{1}^{2 p-1} d_{2 p}^{\prime \prime} \\
& \times\left(\begin{array}{c}
\beta_{1} \\
-\beta_{2} \\
\vdots \\
(-1)^{p+1} \beta_{p}
\end{array}\right)=0
\end{aligned}
$$

and if $n=2 p+1$ to the linear homogeneous system

$$
\begin{aligned}
& \left(\begin{array}{lll}
\omega_{1} d_{1}^{\prime \prime}-\omega_{2} b_{1 p} & \omega_{1}^{3} d_{1}^{\prime \prime}-\omega_{2}^{3} b_{1 p} & \ldots \omega_{1}^{2 p-1} d_{1}^{\prime \prime}-\omega_{2}^{2 p-1} b_{1 p} \\
\omega_{1} d_{2}^{\prime \prime}-\omega_{3} b_{1, p+1} & \omega_{1}^{3} d_{2}^{\prime \prime}-\omega_{3}^{3} b_{1, p+1} & \ldots \omega_{1}^{2 p-1} d_{2}^{\prime \prime}-\omega_{3}^{2 p-1} b_{1, p+1} \\
\ldots & \ldots & \ldots \omega^{2 p-1} d^{\prime \prime} \\
\omega_{1} d_{p-1}^{\prime \prime}-\omega_{p} b_{1,2 p-2} & \omega_{1}^{3} d_{p-1}^{\prime \prime}-\omega_{p}^{3} b_{1,2 p-2} & \ldots \omega_{1}^{2 p-1} b_{1,2 p-2} \\
\omega_{1} d_{p}^{\prime \prime}+\omega_{2} b_{11} & \omega_{1}^{3} d_{p}^{\prime \prime}+\omega_{2}^{3} b_{11} & \ldots \omega_{1}^{2 p-1} d_{p}^{\prime \prime}+\omega_{2}^{2 p-1} b_{11} \\
\omega_{1} d_{p+1}^{\prime \prime}+\omega_{3} b_{12} & \omega_{1}^{3} d_{p+1}^{\prime \prime}+\omega_{3}^{3} b_{12} & \ldots \omega_{1}^{2 p-1} d_{p+1}^{\prime \prime}+\omega_{3}^{2 p-1} b_{12} \\
\ldots & \ldots & \ldots \omega^{2 p-1} d_{2 p-2}^{\prime \prime}+\omega_{p}^{2 p-1} b_{1, p-1} \\
\omega_{1} d_{2 p-2}^{\prime \prime}+\omega_{p} b_{1, p-1} & \omega_{1}^{3} d_{2 p-2}^{\prime \prime}+\omega_{p}^{3} b_{1, p-1} \ldots \omega_{1}^{2 p-1} d_{2 p-1}^{\prime \prime} \\
\omega_{1} d_{2 p-1}^{\prime \prime} & \omega_{1}^{3} d_{2 p-1}^{\prime \prime} & \ldots \omega_{1}^{2 p-1} d_{2 p-1}^{\prime \prime}
\end{array}\right) \\
& \times\left(\begin{array}{c}
\beta_{1} \\
-\beta_{2} \\
\vdots \\
(-1)^{p+1} \beta_{p}
\end{array}\right)=0,
\end{aligned}
$$

where $d_{i}^{\prime \prime}:=-a_{2} d_{1 i}^{\prime}+a_{1} d_{2 i}^{\prime}$. Note that the big matrices have $p$ columns and $2 p-2$, respectively $2 p-1$, rows. For the proof, it suffices to show that the $p \times p$ determinant

$$
\left|\begin{array}{lll}
\omega_{1} d_{1}^{\prime \prime}-\omega_{2} b_{1 p} & \omega_{1}^{3} d_{1}^{\prime \prime}-\omega_{2}^{3} b_{1 p} & \ldots \omega_{1}^{2 p-1} d_{1}^{\prime \prime}-\omega_{2}^{2 p-1} b_{1 p} \\
\omega_{1} d_{2}^{\prime \prime}-\omega_{3} b_{1, p+1} & \omega_{1}^{3} d_{2}^{\prime \prime}-\omega_{3}^{3} b_{1, p+1} & \ldots \omega_{1}^{2 p-1} d_{2}^{\prime \prime}-\omega_{3}^{2 p-1} b_{1, p+1} \\
\ldots & \ldots & \ldots \ldots \\
\omega_{1} d_{p-1}^{\prime \prime}-\omega_{p} b_{1,2 p-2} & \omega_{1}^{3} d_{p-1}^{\prime \prime}-\omega_{p}^{3} b_{1,2 p-2} & \ldots \omega_{1}^{2 p-1} d_{p-1}^{\prime \prime}-\omega_{p}^{2 p-1} b_{1,2 p-2} \\
\omega_{1} d_{p}^{\prime \prime}+\omega_{2} b_{11} & \omega_{1}^{3} d_{p}^{\prime \prime}+\omega_{2}^{3} b_{11} & \ldots \omega_{1}^{2 p-1} d_{p}^{\prime \prime}+\omega_{2}^{2 p-1} b_{11}
\end{array}\right|
$$


does not vanish for generic values of $b_{1 j}$. Operating on the columns gives the determinant

$$
\left|\begin{array}{lll}
\omega_{1} d_{1}^{\prime \prime}-\omega_{2} b_{1 p} & \left(-\omega_{2}^{3}+\omega_{2} \omega_{1}^{2}\right) b_{1 p} & \cdots\left(-\omega_{2}^{2 p-1}+\omega_{2}^{2 p-3} \omega_{1}^{2}\right) b_{1 p} \\
\omega_{1} d_{2}^{\prime \prime}-\omega_{3} b_{1, p+1} & \left(-\omega_{3}^{3}+\omega_{3} \omega_{1}^{2}\right) b_{1, p+1} & \ldots\left(-\omega_{3}^{2 p-1}+\omega_{3}^{2 p-3} \omega_{1}^{2}\right) b_{1, p+1} \\
\cdots & \ldots & \cdots \cdots \\
\omega_{1} d_{p-1}^{\prime \prime}-\omega_{p} b_{1,2 p-2} & \left(-\omega_{p}^{3}+\omega_{p} \omega_{1}^{2}\right) b_{1,2 p-2} & \cdots\left(-\omega_{p}^{2 p-1}+\omega_{p}^{2 p-3} \omega_{1}^{2}\right) b_{1,2 p-2} \\
\omega_{1} d_{p}^{\prime \prime}+\omega_{2} b_{11} & -\left(-\omega_{2}^{3}+\omega_{2} \omega_{1}^{2}\right) b_{11} & \ldots-\left(-\omega_{2}^{2 p-1}+\omega_{2}^{2 p-3} \omega_{1}^{2}\right) b_{11}
\end{array}\right|
$$

and, assuming that $b_{1 p} \neq 0$ and operating on the rows, yields

$$
\begin{aligned}
& \left|\begin{array}{lll}
\omega_{1} d_{1}^{\prime \prime}-\omega_{2} b_{1 p} & \left(-\omega_{2}^{3}+\omega_{2} \omega_{1}^{2}\right) b_{1 p} & \ldots\left(-\omega_{2}^{2 p-1}+\omega_{2}^{2 p-3} \omega_{1}^{2}\right) b_{1 p} \\
\omega_{1} d_{2}^{\prime \prime}-\omega_{3} b_{1, p+1} & \left(-\omega_{3}^{3}+\omega_{3} \omega_{1}^{2}\right) b_{1, p+1} & \ldots\left(-\omega_{3}^{2 p-1}+\omega_{3}^{2 p-3} \omega_{1}^{2}\right) b_{1, p+1} \\
\ldots & \ldots & \ldots \ldots \\
\omega_{1} d_{p-1}^{\prime \prime}-\omega_{p} b_{1,2 p-2} & \left(-\omega_{p}^{3}+\omega_{p} \omega_{1}^{2}\right) b_{1,2 p-2} & \ldots\left(-\omega_{p}^{2 p-1}+\omega_{p}^{2 p-3} \omega_{1}^{2}\right) b_{1,2 p-2} \\
\omega_{1}\left(d_{1}^{\prime \prime} \frac{b_{11}}{b_{1 p}}+d_{p}^{\prime \prime}\right) & 0 & \ldots 0
\end{array}\right| \\
& =(-1)^{p+1} \omega_{1}\left(d_{1}^{\prime \prime} \frac{b_{11}}{b_{1 p}}+d_{1}^{\prime \prime}\right)\left|\begin{array}{ll}
\left(-\omega_{2}^{3}+\omega_{2} \omega_{1}^{2}\right) b_{1 p} & \ldots\left(-\omega_{2}^{2 p-1}+\omega_{2}^{2 p-3} \omega_{1}^{2}\right) b_{1 p} \\
\left(-\omega_{3}^{3}+\omega_{3} \omega_{1}^{2}\right) b_{1, p+1} & \ldots\left(-\omega_{3}^{2 p-1}+\omega_{3}^{2 p-3} \omega_{1}^{2}\right) b_{1, p+1} \\
\ldots & \ldots \ldots \\
\left(-\omega_{p}^{3}+\omega_{p} \omega_{1}^{2}\right) b_{1,2 p-2} & \ldots\left(-\omega_{p}^{2 p-1}+\omega_{p}^{2 p-3} \omega_{1}^{2}\right) b_{1,2 p-2}
\end{array}\right| \\
& =(-1)^{p+1} \omega_{1}\left(d_{1}^{\prime \prime} \frac{b_{11}}{b_{1 p}}+d_{1}^{\prime \prime}\right) b_{1 p} \cdots b_{1,2 p-2}\left|\begin{array}{l}
-\omega_{2}^{3}+\omega_{2} \omega_{1}^{2} \ldots-\omega_{2}^{2 p-1}+\omega_{2}^{2 p-3} \omega_{1}^{2} \\
-\omega_{3}^{3}+\omega_{3} \omega_{1}^{2} \ldots-\omega_{3}^{2 p-1}+\omega_{3}^{2 p-3} \omega_{1}^{2} \\
\cdots \\
-\omega_{p}^{3}+\omega_{p} \omega_{1}^{2} \ldots-\omega_{p}^{2 p-1}+\omega_{p}^{2 p-3} \omega_{1}^{2}
\end{array}\right| \\
& =\omega_{1}\left(d_{1}^{\prime \prime} \frac{b_{11}}{b_{1 p}}+d_{1}^{\prime \prime}\right) b_{1 p} \cdots b_{1,2 p-2}\left(\omega_{2}^{3}-\omega_{2} \omega_{1}^{2}\right) \cdots\left(\omega_{p}^{3}-\omega_{p} \omega_{1}^{2}\right)\left|\begin{array}{cccc}
1 & \omega_{2}^{2} & \ldots & \omega_{2}^{2(p-2)} \\
1 & \omega_{3}^{2} \ldots . \omega_{3}^{2(p-2)} \\
\ldots & \ldots \ldots \\
1 & \omega_{p}^{2} \ldots \omega_{p}^{2(p-2)}
\end{array}\right| \\
& =\omega_{1} \cdots \omega_{p}\left(\omega_{2}^{2}-\omega_{1}^{2}\right) \cdots\left(\omega_{p}^{2}-\omega_{1}^{2}\right) \prod_{2 \leq i<j \leq p}\left(\omega_{j}^{2}-\omega_{i}^{2}\right)\left(d_{1}^{\prime \prime} b_{11}+d_{1}^{\prime \prime} b_{1 p}\right) b_{1, p+1} \cdots b_{1,2 p-2} .
\end{aligned}
$$

Because of the generic choice $0<\omega_{1}<\cdots<\omega_{p}$, all factors involving the $\omega_{k}$ are strictly positive. Thus, assuming the generic conditions $b_{1 j} \neq 0$ for all $j=p, \ldots, 2 p-2$ and $d_{1}^{\prime \prime} b_{11}+d_{1}^{\prime \prime} b_{1 p} \neq 0$, this determinant does not vanish.

This proves that the functions $H_{1}, \ldots, H_{p}$ are independent.

4.3 Independence of the Functions $H_{1}, \ldots, H_{p}, \delta_{1}, \ldots \delta_{p-1}$ if $n=2 p+1$ and $H_{1}, \ldots, H_{p-1}, \delta_{1}, \ldots \delta_{p-1}$ if $n=2 p$

We begin with the case $n=2 p+1$ and show that $H_{1}, \ldots, H_{p}, \delta_{1}, \ldots \delta_{p-1}$ are independent functions. As we shall see, we shall use in this proof the separate independence of the sets of functions $\left\{\delta_{1}, \ldots, \delta_{p-1}\right\}$ and $\left\{H_{1}, \ldots, H_{p}\right\}$ proved so far. Suppose that

$$
\beta_{1} \frac{\partial H_{1}}{\partial \vec{p}}+\beta_{2} \frac{\partial H_{2}}{\partial \vec{p}}+\cdots+\beta_{p} \frac{\partial H_{p}}{\partial \vec{p}}+\alpha_{1} \frac{\partial \delta_{1}}{\partial \vec{p}}+\alpha_{2} \frac{\partial \delta_{2}}{\partial \vec{p}}+\cdots+\alpha_{p-1} \frac{\partial \delta_{p-1}}{\partial \vec{p}}=0,
$$


where $\frac{\partial H_{k}}{\partial \vec{p}}$ is given by formula (4.13) and $\frac{\partial \delta_{1}}{\partial \vec{p}}$ by formula (4.2). This is a $(2 p-1) \times(2 p-1)$ linear homogeneous system with unknowns $\beta_{1},-\beta_{2}, \beta_{3}, \ldots$, $(-1)^{p-1} \beta_{p}, \alpha_{1},-\alpha_{2}, \alpha_{3}, \ldots,(-1)^{p} \alpha_{p-1}$ given by the matrix

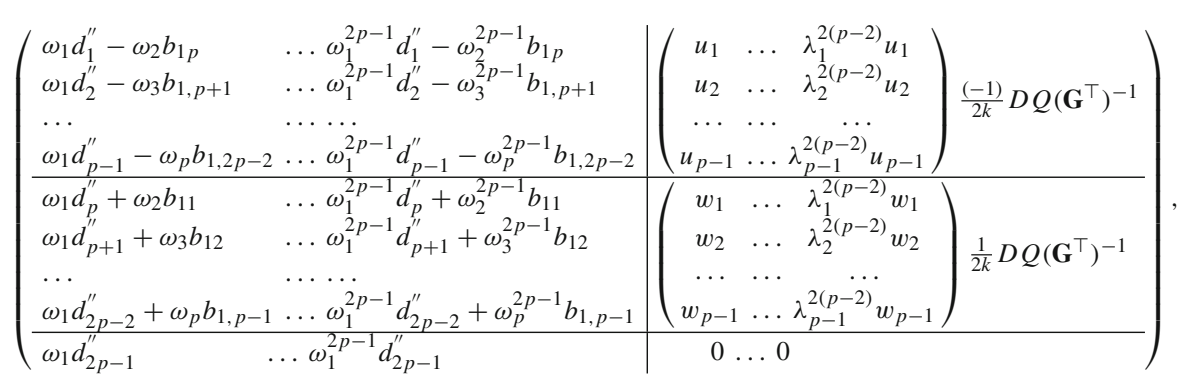

where $D, Q$ are introduced in (4.3) and $\mathbf{G}$ in (4.14). We must show that its determinant does not vanish, generically. Operating on the columns and rows yields

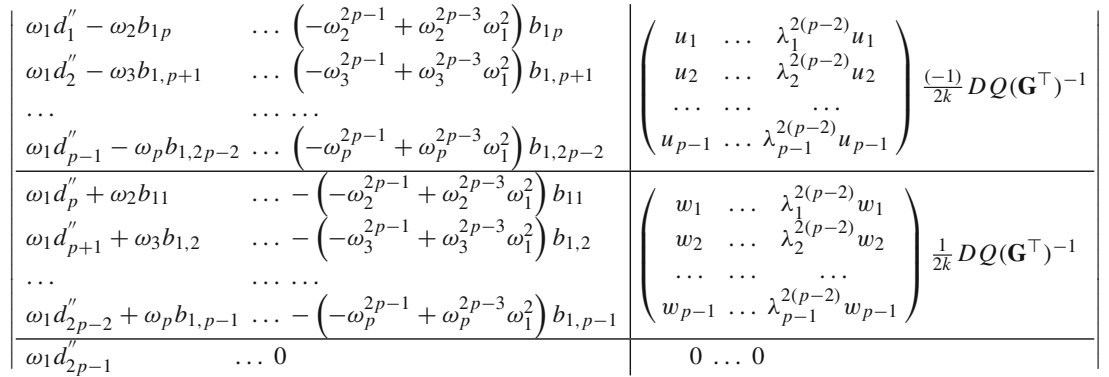

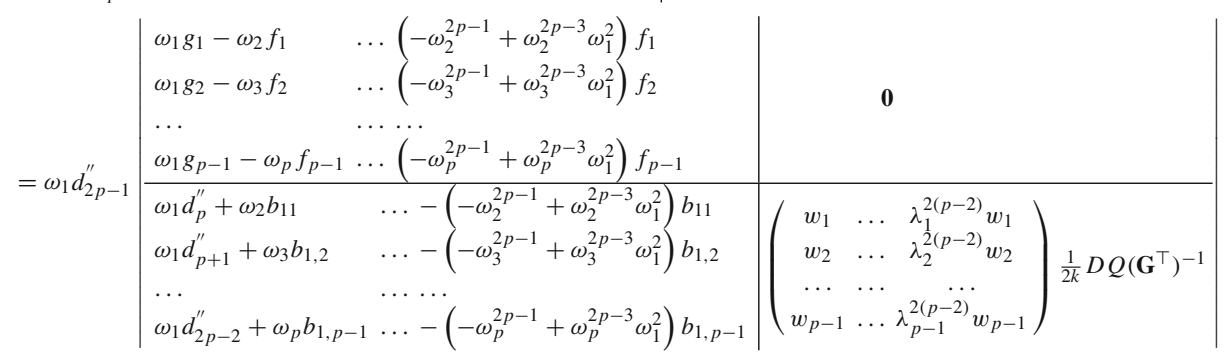

$$
\begin{aligned}
& =\omega_{1} d_{2 p-1}^{\prime \prime}\left|\begin{array}{ll}
\omega_{1} g_{1}-\omega_{2} f_{1} & \ldots\left(-\omega_{2}^{2 p-1}+\omega_{2}^{2 p-3} \omega_{1}^{2}\right) f_{1} \\
\omega_{1} g_{2}-\omega_{3} f_{2} & \ldots\left(-\omega_{3}^{2 p-1}+\omega_{3}^{2 p-3} \omega_{1}^{2}\right) f_{2} \\
\ldots & \ldots \ldots
\end{array}\right|\left|\begin{array}{ccc}
w_{1} & \ldots & \lambda_{1}^{2(p-2)} w_{1} \\
w_{2} & \ldots & \lambda_{2}^{2(p-2)} w_{2} \\
\ldots & \ldots & \ldots \\
w_{p-1} & \ldots & \lambda_{p-1}^{2(p-2)} w_{p-1}
\end{array}\right|\left|\frac{1}{2 k} D Q\left(\mathbf{G}^{\top}\right)^{-1}\right|,
\end{aligned}
$$

where $g_{i}=d_{i}^{\prime \prime}+\frac{u_{i}}{w_{i}} d_{p+i-1}^{\prime \prime}, f_{i}=b_{1, p+i-1}-\frac{u_{i}}{w_{i}} b_{1, i}, i=1, \ldots, p-1$ and we required the generic condition that all $w_{i} \neq 0$. These types of determinants were calculated in the previous two special cases and shown that they do not vanish, generically.

If $n=2 p$, the independence of the functions $H_{1}, \ldots, H_{p-1}, \delta_{1}, \ldots, \delta_{p-1}$ is shown in a similar manner. The relevant determinant is now 


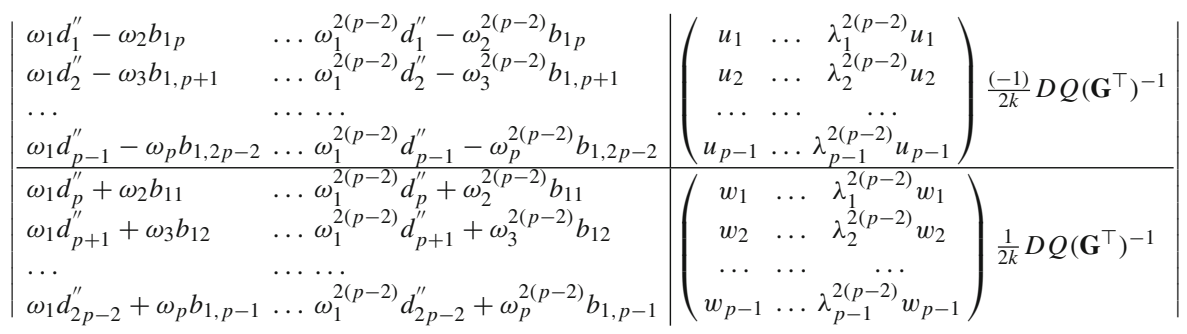

and the proof proceeds following analogous steps as in the case $n=2 p+1$.

Note that there is no contradiction of the independence of the complete set of functions $\left\{H_{1}, \ldots, H_{p-1}, \delta_{1}, \ldots \delta_{p-1}\right\}$ with the fact that the two sets of functions $\left\{H_{1}, \ldots, H_{p}\right\}$ and $\left\{\delta_{1}, \ldots \delta_{p-1}\right\}$ are separately independent. As pointed out in examples, $H_{p}$ and the Pfaffian are both expressible in terms of the other integrals.

Theorem 4.1 The collection of functions $\left\{H_{1}, \ldots, H_{p}, \delta_{1}, \ldots \delta_{p-1}\right\}$ if $n=2 p+1$ and $\left\{H_{1}, \ldots, H_{p-1}, \delta_{1}, \ldots \delta_{p-1}\right\}$ if $n=2 p$ is an integrable system on the $2(n-2)$-dimensional vector space $\operatorname{Mat}_{2 \times(n-2)}(\mathbb{R})$ endowed with the canonical symplectic structure.

The case for general $\epsilon \in \mathbb{R}$ is treated in a similar manner. The proofs are identical but the writing is more cumbersome.

Theorem 4.2 The collection of functions $\left\{H_{1, \epsilon}, \ldots, H_{p, \epsilon}, \delta_{1}, \ldots, \delta_{p-1}\right\}$ if $n=2 p+1$ and $\left\{H_{1, \epsilon}, \ldots, H_{p-1, \epsilon}, \delta_{1}, \ldots, \delta_{p-1}\right\}$ if $n=2 p$ is an integrable system on the $2(n-2)$ dimensional vector space $\operatorname{Mat}_{2 \times(n-2)}(\mathbb{R})$ endowed with the canonical symplectic structure.

Acknowledgments Alina Dobrogowska was supported by Swiss SCIEX grant 10.246 POL and Tudor S. Ratiu was partially supported by Swiss NSF grant 200021-140238, and by the government grant of the Russian Federation for support of research projects implemented by leading scientists, Lomonosov Moscow State University under the agreement No. 11.G34.31.0054.

\section{References}

1. Abraham, R., Marsden, J.E.: Foundations of Mechanics, 2nd edn., revised and enlarged. With the assistance of Tudor Ratiu and Richard Cushman. Benjamin/Cummings Publishing Co., Inc., Advanced Book Program, Reading, MA (1978)

2. Adler, M.: On a trace functional for formal pseudo differential operators and the symplectic structure of the Korteweg-de Vries type equations. Invent. Math. 50(3), 219-248 (1978/79)

3. Adler, M., van Moerbeke, P., Vanhaecke, P.: Algebraic Integrability, Painlevé Geometry and Lie Algebras, Ergebnisse der Mathematik und ihrer Grenzgebiete, 3. Folge, vol. 47. Springer, Berlin (2004)

4. Arnold, V.I.: Mathematical Methods of Classical Mechanics, 2nd ed., Graduate Texts in Mathematics, vol. 60. Springer, New York (1989)

5. Arnold, V.I., Kozlov, V.V., Neishtadt, A.I.: Mathematical Aspects of Classical and Celestial Mechanics (Dynamical Systems III), 3rd edn. Encyclopaedia of Mathematical Sciences, vol. 3. Springer, Berlin (2006)

6. Guillemin, V., Sternberg, S.: On collective complete integrability according to the method of Thimm. Ergod. Theory Dyn. Syst. 3(2), 219-230 (1983)

7. Kostant, B.: The solution to a generalized Toda lattice and representation theory. Adv. Math. 34(3), 195-338 (1979)

8. Kazhdan, D., Kostant, B., Sternberg, S.: Hamiltonian group actions and dynamical systems of Calogero type. Commun. Pure Appl. Math. 31(4), 481-507 (1978)

9. Magri, F.: A simple model of the integrable Hamiltonian equation. J. Math. Phys. 19(5), 1156-1162 (1978) 
10. Mishchenko, A.S., Fomenko, A.T.: Euler equation on finite-dimensional Lie groups (Russian). Izv. Akad. Nauk SSSR Ser. Mat. 42(2), 396-415, 471 (1978)

11. Mishchenko, A.S., Fomenko, A.T.: Integrability of Euler's equations on semisimple Lie algebras (Russian). Trudy Sem. Vektor. Tenzor. Anal. 19, 3-94 (1979)

12. Neumann, C.: De problemate quodam mechanica, quod ad primam integralium ultra-ellipticorum classem revocatur. J. Reine u. Angew. Math. 56, 54-66 (1859)

13. Odzijewicz, A., Dobrogowska, A.: Integrable Hamiltonian systems related to the Hilbert-Schmidt ideal. J. Geom. Phys. 61, 1426-1445 (2011)

14. Odzijewicz, A., Goliński, T.: Hierarchy of integrable Hamiltonians describing the nonlinear $n$-wave interaction. J. Phys. A 45(4), 045204 (2012)

15. Ratiu, T.S.: The, C. Neumann problem as a completely integrable system on an adjoint orbit. Trans. Am. Math. Soc. 264(2), 321-329 (1981)

16. Reyman, A.G., Semenov-Tian-Shansky, M.A.: Group-theoretical methods in the theory of finitedimensional integrable systems. In: Dynamical Systems. VII. Integrable Systems, Nonholonomic Dynamical Systems. Encyclopaedia of Mathematical Sciences, vol. 16. Springer, Berlin (1994)

17. Semenov-Tian-Shansky, M.A.: What is a classical r-matrix? Funct. Anal. Appl. 17(4), 259-272 (1983)

18. Symes, W.W.: Hamiltonian group actions and integrable systems. Phys. D 1(4), 339-374 (1980)

19. Thimm, A.: Integrable geodesic flows on homogeneous spaces. Ergod. Theory Dyn. Syst. 1(4) (1981), 495-517 (1982) 